\title{
Displacement modes of a thin-walled beam model with deformable cross sections
}

\author{
Hansen, Anders Bau; Jönsson, Jeppe
}

Published in:

Thin-Walled Structures

Link to article, DOI:

10.1016/j.tws.2019.01.052

Publication date:

2019

Document Version

Peer reviewed version

Link back to DTU Orbit

Citation (APA):

Hansen, A. B., \& Jönsson, J. (2019). Displacement modes of a thin-walled beam model with deformable cross sections. Thin-Walled Structures, 141, 576-592. https://doi.org/10.1016/j.tws.2019.01.052

\section{General rights}

Copyright and moral rights for the publications made accessible in the public portal are retained by the authors and/or other copyright owners and it is a condition of accessing publications that users recognise and abide by the legal requirements associated with these rights.

- Users may download and print one copy of any publication from the public portal for the purpose of private study or research.

- You may not further distribute the material or use it for any profit-making activity or commercial gain

- You may freely distribute the URL identifying the publication in the public portal

If you believe that this document breaches copyright please contact us providing details, and we will remove access to the work immediately and investigate your claim. 


\title{
Displacement Modes of a Thin-Walled Beam Model with Deformable Cross Sections
}

\author{
Anders Bau Hansen ${ }^{\mathrm{a}, \mathrm{b}}$, Jeppe Jönsson ${ }^{\mathrm{a}, *}$ \\ ${ }^{a}$ Technical University of Denmark, Department of Civil Engineering, Brovej Building 118, DK-2800 Kgs. Lyngby, Denmark \\ ${ }^{b}$ NIRAS A/S, Sortemosevej 19, DK-3450 Allerød, Denmark
}

\begin{abstract}
A novel one dimensional beam model for analysis of prismatic thin-walled beams with deformable cross sections is introduced and a novel cross section mode determination procedure, which leads to the three dimensional beam displacement modes, is derived. The first order beam model for linear analysis includes: shear deformations related to both Timoshenko and Mindlin-Reissner type shear deformations, the warping effects of torsion, cross section distortion with related warping effects, as well as the Poisson effect with transverse displacements due to normal stress. The generality of the model allows it to handle open, closed and multi-cell cross sections with branched walls. The cross section analysis procedure leads to two types of beam displacement modes referred to as distortional beam modes and fundamental beam modes, with exponential and polynomial variations along the beam axis, respectively. It turns out that each of the beam deformation modes consists of a sum of one to four cross section displacement fields each with an individual axial variation. The displacement modes can facilitate the formulation of an advanced thin-walled beam element. The beam displacement modes will be illustrated for an open and a closed cross section.
\end{abstract}

Keywords: Thin-walled beams, Beam eigenvalue problem, Warping, Distortion, Shear deformations, Fundamental beam modes, Beam theory

\section{Introduction}

Through centuries, beam models have been developed and especially in the twentieth century the development of thin-walled models increased due to the emerging ship and aircraft industries. It was realized that theories, such as the Euler-Bernoulli and the Timoshenko theories were not sufficient for assessing thin-walled structures even when unconstrained uniform St. Venant torsion was included. A reason was the missing cross sectional warping effect, thus, new theories had to be derived. The best known consistent theory is the torsional thin-walled beam theory presented by Vlasov [1]. This theory includes the warping phenomenon by introducing the sectorial coordinate. With this new "coordinate", the axial displacement field of the cross section is defined as a warping function. With this warping function, a decoupling of the displacement field into the classic extension and flexural modes is still possible, similar to earlier classic beam theories. However, Vlasov's theory of torsion does not include distortion of the cross section, since the cross section is assumed to maintain its shape. A generalization of Vlasov's thin-walled beam theory for open cross sections including distortion was given by Schardt [2]. Later, Schardt extended the theory to include closed cross sections as well, 33. To do this he relaxed the Vlasov hypothesis of negligible shear

\footnotetext{
* Corresponding author

Email address: jej@byg.dtu.dk (Jeppe Jönsson)
}

strain adopting "Bredt's shear flow" in closed cells. At first, Schardt's theory was named: "Verallgemeinerte Technishe Biegetheorie" (VTB), but with its international spread, due to the work done by Davies and co-workers [4, 5], it is now known as GBT, i.e. Generalized Beam Theory, in the international academic society.

An expansion of Vlasovs thin-walled beam theory to include a single distortional deformation mode, which is directly applicable to both open and closed cross sections, was given by Jönsson in [6]. This reference illustrates the coupling of the differential equations, which are not easily uncoupled even in this relatively simple theory.

In recent years Silvestre and Camotim contributed heavily to the development and refinement of GBT and they also include orthotropic material behaviour [7, 8].

The general idea of modern GBT is to use a cross section discretization to determine orthogonal cross section displacement modes, i.e. displacement fields of a cross section. These modes are then interpolated using cubic Hermite functions in the axial direction. The cross section displacement modes are found from solving special eigenvalue problems related to equilibrium equations of the cross section using frame analogy. This approach, deriving displacement modes based on a discretized cross section, has become popular among researchers, since only a relatively small number of unknowns are needed. Modern GBT establishes a thin-walled beam model through rationalized and automated cross section analysis procedures and numerical analysis, see for example Bebiano et al. 9] 
and recently GBT has been extended to curved members by Peres et al. 10 .

The "Finite Strip Method" (FSM) is another very popular method used to model the behaviour of thin-walled beams with deformable cross sections. With the refined "constrained Finite Strip Method" (cFSM) developed by Ádány and Schafer [11, it is possible to group the modes into categories; local modes, distortional mode, and beam modes as already done using GBT. A comparison of these two methods can be found in Ádány et al. [12].

The "constrained Finite Element Method" (CFEM) in which shell finite elements are used to model thin-walled beams by constraining displacements may also be used to create approximate beam models. The accuracy of these models depend on the underlying discretization in both transverse and longitudinal directions as well as on the constraints adapted. The constraints reduce the number of degrees of freedom and lead to advanced thin-walled beam elements, see for example Ádány [13].

Other generalized beam models based on discretization of the cross section adapt a more strict mathematical approach by formulating and solving the coupled equilibrium equations, i.e. they adapt the strong solution form. See for example the appoaches by Jönsson and Andreassen [14, Morandini et al. [15] and Vieira et al. [16]. All of these authors solve eigenvalue problems as part of a standard procedure for finding the solution to the coupled homogeneous beam differential equations for arbitrary cross sections. As opposed to GBT, these approaches lead to eigenvectors being the cross section displacement fields multiplied by the exponential eigenfunction determined by the eigenvalues. The polynomial modes (related to the classic beam modes) are derived from a detailed evaluation of the null-eigenvalue modes, hence, two sets of solutions are obtained. Morandini et al. find twelve fundamental beam solutions for solid cross sections using a special procedure based on the Jordan chain method. Furthermore, they find the eigenvalues of the exponential solutions and relate these to length scales as well as the St. Venant principle. Vieira et al. also uses a Jordan chain method to establish the polynomial solution modes, which are identified as fundamental beam modes, e.g. extension, flexure, etc. Furthermore, they use isospectral transformations of exponential modes to define generalized solution modes.

The prismatic thin-walled beam model presented in the following also adheres to the more strict mathematical approach of solving the coupled equilibrium equations. Some of the kinematic assumptions are made in order to facilitate the secondary goal of being able to connect to joints, which are modeled using conventional finite shell elements with three translational and three rotational degrees of freedom at each node. Furthermore it has also been the goal to achieve a formulation including shear deformations and the Poisson effect as opposed to the theoretical model presented in Jönsson and Andreassen [14, which does not include these effects. As opposed to Morandini et al. and Vieira et al. the present work retains the complex eigen- values and exponential solution modes of the quadratic eigenvalue problem and therefore the solutions do not diagonalize all matrices of the original coupled differential equilibrium equations of the beam, since this is unnecessary as the modes span the solution space.

In this paper, the exact solution modes of the coupled differential beam equations are found through cross section discretization and separation of variables into cross section displacement fields and attenuation functions. The solutions to the differential equations leads to solutions consisting of fundamental polynomial beam solutions modes and exponential distortional solution modes (decaying from each beam end). The solution modes involve from one to four cross section displacement fields combined into a single three-dimensional solution mode being either polynomial or exponential. The advantage of this beam model is that the full solution is obtained between beam-ends and not as in GBT in which a beam must be discretized into several GBT finite elements with Hermitian interpolation along the axis of the beam.

\section{The thin-walled beam model}

The prismatic one dimensional thin-walled beam model is introduced in the following subsections. It is assumed that the beam displacements can be subdivided into a sum of separate beam displacement modes, which all obey the equilibrium equations. In the first subsection the kinematics and strains of one displacement mode is introduced and illustrated. Then, in the second subsection we introduce the strain energy function using the introduced displacement assumptions. Hereafter in the third subsection the cross section is discretized into straight wall elements and interpolation functions and related degrees of freedom are introduced and chosen. The fourth subsection introduces the discretization into the strain energy, which leads to the definition of wall element stiffness matrices, which in turn assemble into the stiffness matrices of the cross section. In the last and fifth subsection the variation of the discretized strain energy leads to the strong formulation of displacement mode equilibrium as a set of coupled second order differential equations expressed by the cross sectional stiffness matrices.

\subsection{Kinematics}

The prismatic thin-walled beam member is located in a global rectangular, right-handed Cartesian coordinate system $(X, Y, Z)$. The $Z$-axis is pointing in the longitudinal direction of the member and the cross section is located in the $X, Y$-plane. To navigate through the cross section a local coordinate system is introduced in the walls as $(n, s, z)$ following the right-hand convention and where $z$ is parallel to the global $Z$-axis. The $s$-coordinate is a curve parameter running along the center line of the cross section and $n$ indicates the normal to the $s, z$-plane. In figure 1 both local $(n, s, z)$ and global $(X, Y, Z)$ coordinate systems are 
illustrated. The notation used in the following for derivatives with respect to the cross section wall coordinates will be a subscript comma followed by the local coordinate, e.g. as: $(\cdot)_{, n} \equiv d(\cdot) / d n$ or $(\cdot)_{, s s} \equiv d^{2}(\cdot) / d s^{2}$; however, derivatives with respect to the axial coordinate will be denoted by a prime, i.e. $(\cdot)^{\prime} \equiv d(\cdot) / d z$.

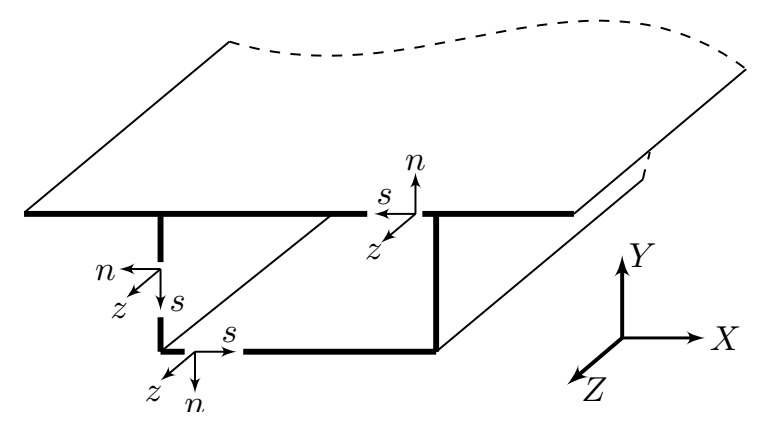

Figure 1: Illustration of local and global coordinate systems

Beam displacement modes are introduced by separation of variables through the sum of products between displacement variables dependent on the cross section coordinates $(n, s)$ and amplitude functions dependent on the axial coordinate $z$. The cross section displacements are given in the local coordinate directions as functions of the local coordinates $(n, s)$ and the axial coordinate $z$. The displacements of one displacement mode thus consists of transverse displacements $\left(u_{n}, u_{s}\right)$ and (axial) warping displacements $u_{z}$. The kinematic displacement parameters are illustrated in figure 2

Let us start by introducing the transverse displacements $\left(u_{n}, u_{s}\right)$ of one displacement mode as:

$$
\begin{aligned}
& u_{n}(s, z)=w_{n}(s) \psi(z) \\
& u_{s}(n, s, z)=\left[w_{s}(s)-n w_{n, s}(s)\right] \psi(z)
\end{aligned}
$$

in which $w_{n}(s)$ and $w_{s}(s)$ are the center line displacements in local directions factorized by a function of the axial coordinate, $\psi(z)$, referred to as an axial amplitude function. This displacement formulation corresponds to that of thin-plate theory or Kirchhoff theory in the transverse $s$-direction. This assumption will in a later subsection allow us to use beam like wall elements with a traditional beam type interpolation.

Then, let us introduce the axial warping displacements $u_{z}$ of the displacement mode as:

$$
u_{z}(n, s, z)=[\Omega(s)+n \alpha(s)] \eta(z)
$$

where $\Omega(s)$ is the axial displacement of the wall center line, $\alpha(s)$ is the additional inclination through the thickness, and $\eta(z)$ is the related amplitude function, see figure 2. This formulation corresponds to a Timoshenko like shear formulation in the planes of each wall element $(s, z$-plane) and a Mindlin-Reisner like shear formulation through the wall element thickness in the axial $z$-direction (n,z-plane). This warping displacement formulation has been chosen to allow a strict local warping compatibility at the corners under the hypothesis that this would allow an enhanced modeling of shear, see for example the related GBT type assumptions and illustrations in Miranda et al. [17] and [18. In a later subsection these assumptions also allow the introduction of six degrees of freedom per node in the cross section interpolation. Furthermore, the kinematic displacement assumptions also allow torsional shear flow around closed cells as considered by Jönsson and Andreassen [14].

In the elastic first order model, the linear strain definitions are applicable and they can be expressed by the introduced displacement description as follows:

$$
\begin{array}{lc}
\varepsilon_{s s}=u_{s, s} & =\left(w_{s, s}-n w_{n, s s}\right) \psi \\
\varepsilon_{z z}=u_{z}^{\prime} & =(\Omega+n \alpha) \eta^{\prime} \\
\gamma_{z s}=u_{s}^{\prime}+u_{z, s} & =\left(w_{s}-n w_{n, s}\right) \psi^{\prime} \\
& \quad+\left(\Omega_{, s}+n \alpha_{, s}\right) \eta \\
\gamma_{z n}=u_{n}^{\prime}+u_{z, n} & =w_{n} \psi^{\prime}+\alpha \eta
\end{array}
$$

where $\varepsilon_{s s}$ are the transverse distortional strains, $\varepsilon_{z z}$ are axial normal strains, $\gamma_{z s}$ are the membrane shear strains of the wall, and $\gamma_{z n}$ are the through-wall axial shear strains. It follows that $\varepsilon_{n n}=0$ and $\gamma_{s n}=0$. It is seen that, due to the displacement formulations, we achieve non-null $\gamma_{z n}$-strains. It is also seen that the kinematics presented here includes transverse extension along the cross section mid-line as well as membrane shear strain, which both are neglected in the Vlasov theory (the Vlasov hypothesis).

In the following, vectors and matrices will be denoted by non-slanted, boldfaced, roman letters.

\subsection{Discretization and interpolation of strain energy}

The constitutive relations between stresses and strains are not as simple as in conventional beam theories, since the theory not only includes axial stresses $\sigma_{z z}$ but also transverse stresses $\sigma_{s s}$, membrane wall shear stresses $\tau_{s z}$, as well as transverse wall shear stresses $\tau_{n z}$. As in plate theory we base the constitutive relation of the wall on the assumption of zero out of plane normal stress, i.e. $\sigma_{n n}=0$. The axial and transverse in-plane normal stresses are coupled through the Poisson ratio, $\nu$, and enables the Poisson effect, which induces changes in the geometry of the cross section. Using Young's modulus of elasticity $E$, we introduce the shear modulus $G=E /(2(1+\nu))$ and the plate elasticity modulus $E_{s}=E /\left(1-\nu^{2}\right)$. Hence, the linear constitutive relations are:

$$
\left[\begin{array}{c}
\sigma_{s s} \\
\sigma_{z z} \\
\tau_{s z} \\
\tau_{n z}
\end{array}\right]=\left[\begin{array}{cccc}
E_{s} & \nu E_{s} & 0 & 0 \\
\nu E_{s} & E_{s} & 0 & 0 \\
0 & 0 & G & 0 \\
0 & 0 & 0 & G
\end{array}\right]\left[\begin{array}{c}
\varepsilon_{s s} \\
\varepsilon_{z z} \\
\gamma_{s z} \\
\gamma_{n z}
\end{array}\right]
$$




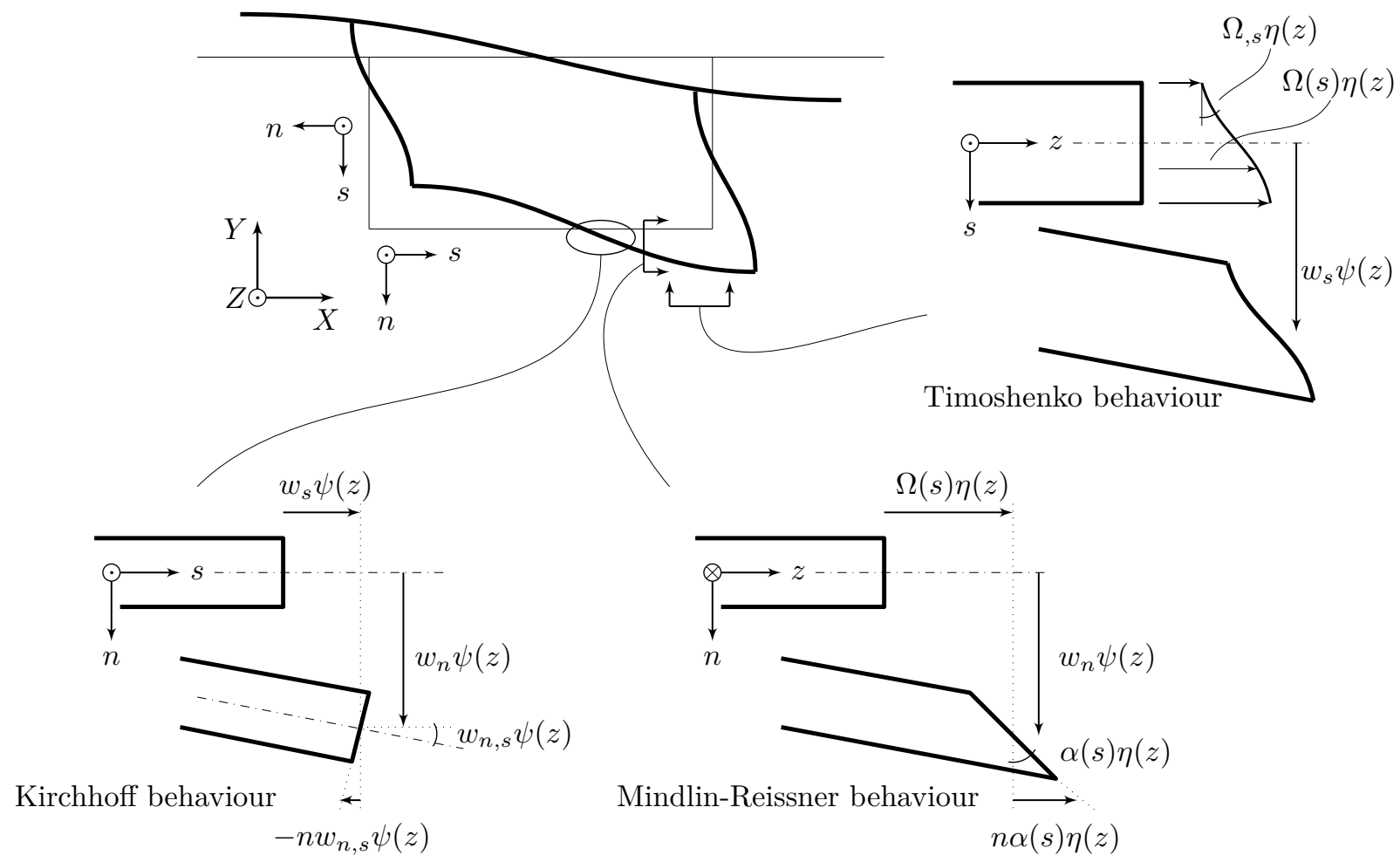

Figure 2: Deformations with respect to the cross section in different orientations

With the introduced constitutive relations, the linear elastic strain energy can be written as follows:

$$
\begin{array}{r}
U=\frac{1}{2} \int_{V}\left\{E_{s} \varepsilon_{s s}^{2}+E_{s} \varepsilon_{z z}^{2}+2 \nu E_{s} \varepsilon_{s s} \varepsilon_{z z}\right. \\
\left.+G \gamma_{n z}^{2}+G \gamma_{s z}^{2}\right\} d V
\end{array}
$$

Let us introduce the strains, equation (4), expressed by the displacement parameters into the strain energy. Then, let us perform the volume integration by subdividing the cross section into a sum of integrals over each straight wall element and let us perform the integration through the thickness. Thus, the middle-surface of a thin-walled cross section is assembled by straight wall elements as illustrated in figure 3 In the current formulation a wall element is characterized by a constant plate thickness $t_{e l}$ and an element length of $b_{e l}$. The strain energy equation (6) for one displacement mode of a beam of length $\ell$ becomes:

$$
\begin{aligned}
U= & \frac{1}{2} \int_{0}^{\ell} \sum_{\mathrm{el}} \int_{0}^{b_{e l}}\left\{E_{s}\left(t_{e l}\left(w_{s, s} \psi\right)^{2}+\frac{1}{12} t_{e l}^{3}\left(w_{n, s s} \psi\right)^{2}\right)\right. \\
+ & E_{s}\left(t_{e l}\left(\Omega \eta^{\prime}\right)^{2}+\frac{1}{12} t_{e l}^{3}\left(\alpha \eta^{\prime}\right)^{2}\right) \\
& +2 \nu E_{s}\left(t_{e l}\left(w_{s, s} \psi \Omega \eta^{\prime}\right)-\frac{1}{12} t_{e l}^{3} w_{n, s s} \psi \alpha \eta^{\prime}\right) \\
& +G\left(t_{e l}\left(w_{s} \psi^{\prime}\right)^{2}+2 t_{e l} w_{s} \psi^{\prime} \Omega, s \eta+\frac{1}{12} t_{e l}^{3}\left(w_{n, s} \psi^{\prime}\right)^{2}\right. \\
& \left.-2 \frac{1}{12} t_{e l}^{3} w_{n, s} \psi^{\prime} \alpha_{, s} \eta+t_{e l}(\Omega, s \eta)^{2}+\frac{1}{12} t_{e l}^{3}(\alpha, s \eta)^{2}\right) \\
& \left.+G\left(t_{e l}\left(w_{n} \psi^{\prime}\right)^{2}+t_{e l}(\alpha \eta)^{2}+2 t_{e l} w_{n} \psi^{\prime} \alpha \eta\right)\right\} d s d z
\end{aligned}
$$

An example of the overall discretization of a cross section is illustrated in figure $3 \mathrm{a}$ and the configuration of a discrete wall element is shown in figure $3 \mathrm{~b}$ illustrating its degrees of freedom. The wall element displacements are derived from approximated linear Lagrange and cubic Hermite functions. The degrees of freedom are split into two - those regarding displacements in the cross sectional plane given a $w$-subscript are transverse displacements, and those regarding displacements out of the cross sectional plane denoted by an $\Omega$ are in general being referred to as warping displacements. Thus, the wall element de- 


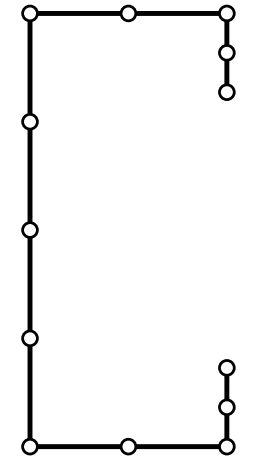

(a) Cross section discretized into minor wall elements

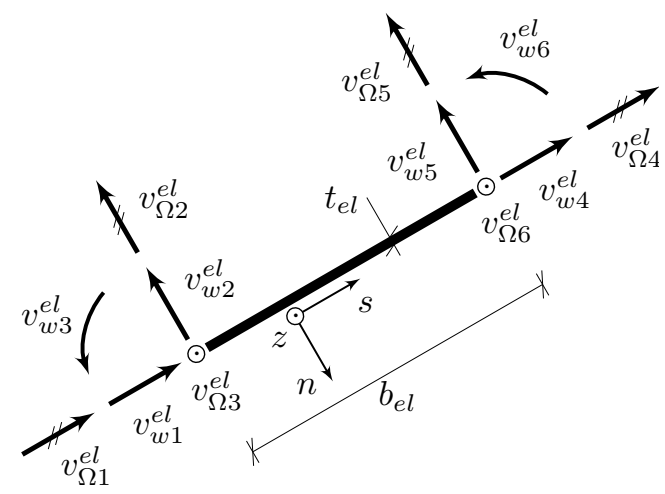

(b) A wall element with its twelve degrees of freedom and local coordinate system

Figure 3: A discretized thin-walled cross section and a wall element

grees of freedom are collected in two vectors being:

$$
\begin{aligned}
& \mathbf{v}_{w}^{e l}=\left[\begin{array}{llllll}
v_{w 1}^{e l} & v_{w 2}^{e l} & v_{w 3}^{e l} & v_{w 4}^{e l} & v_{w 5}^{e l} & v_{w 6}^{e l}
\end{array}\right]^{\top} \\
& \mathbf{v}_{\Omega}^{e l}=\left[\begin{array}{llllll}
v_{\Omega 1}^{e l} & v_{\Omega 2}^{e l} & v_{\Omega 3}^{e l} & v_{\Omega 4}^{e l} & v_{\Omega 5}^{e l} & v_{\Omega 6}^{e l}
\end{array}\right]^{\top}
\end{aligned}
$$

The introduced cross section displacement parameters are interpolated within the cross section using standard interpolation functions as follows (for clarity we have kept the related amplitude functions):

$$
\begin{aligned}
& w_{s} \psi=\mathbf{N}_{s} \mathbf{v}_{w}^{e l} \psi, \quad \alpha \eta=\mathbf{N}_{\alpha} \mathbf{v}_{\Omega}^{e l} \eta \\
& w_{n} \psi=\mathbf{N}_{n} \mathbf{v}_{w}^{e l} \psi, \quad \Omega \eta=\mathbf{N}_{\Omega} \mathbf{v}_{\Omega}^{e l} \eta
\end{aligned}
$$

in which the interpolation vectors are given in table 1 Furthermore, $\mathbf{N}_{s}$ and $\mathbf{N}_{\alpha}$ are linear whereas $\mathbf{N}_{n}$ and $\mathbf{N}_{\Omega}$ are cubic, see [19].

Table 1: Interpolation vectors

$$
\begin{aligned}
& \mathbf{N}_{s}=\left[\begin{array}{llllll}
N_{1} & 0 & 0 & N_{4} & 0 & 0
\end{array}\right] \\
& \mathbf{N}_{n}=\left[\begin{array}{llllll}
0 & -N_{2} & -N_{3} & 0 & -N_{5} & -N_{6}
\end{array}\right] \\
& \mathbf{N}_{\alpha}=\left[\begin{array}{llllll}
-N_{1} & 0 & 0 & -N_{4} & 0 & 0
\end{array}\right] \\
& \mathbf{N}_{\Omega}=\left[\begin{array}{llllll}
0 & -N_{3} & N_{2} & 0 & -N_{6} & N_{5}
\end{array}\right]
\end{aligned}
$$

\subsection{Cross section stiffness matrices}

If the displacement interpolation functions, from equation (9), are substituted into equation (7), local wall element stiffness matrices given in table 2 may be found by integration over the element wall length. Regarding the superscripts, these indicate whether a stiffness matrix relates to transverse, normal or shearing stiffness, denoted: $s, \sigma$ or $\gamma$, respectively. The coupling terms between axial and transverse normal strain are given by $\mathbf{k}_{w \Omega}^{s \sigma}$ and its transpose, which becomes $\mathbf{k}_{\Omega w}^{\sigma s}$.
Table 2: Local stiffness matrices

$$
\begin{aligned}
& \mathbf{k}_{w w}^{s}=\int_{0}^{b_{e l}}\left(t_{e l} E_{s} \mathbf{N}_{s, s}^{\top} \mathbf{N}_{s, s}+\frac{t_{e l}^{3}}{12} E_{s} \mathbf{N}_{n, s s}^{\top} \mathbf{N}_{n, s s}\right) d s \\
& \mathbf{k}_{\Omega \Omega}^{\sigma}=\int_{0}^{b_{e l}}\left(t_{e l} E_{s} \mathbf{N}_{\Omega}^{\top} \mathbf{N}_{\Omega}+\frac{t_{e l}^{3}}{12} E_{s} \mathbf{N}_{\alpha}^{\top} \mathbf{N}_{\alpha}\right) d s \\
& \mathbf{k}_{w \Omega}^{s \sigma}=\int_{0}^{b_{e l}}\left(t_{e l} \nu E_{s} \mathbf{N}_{s, s}^{\top} \mathbf{N}_{\Omega}-\frac{t_{e l}^{3}}{12} \nu E_{s} \mathbf{N}_{n, s s}^{\top} \mathbf{N}_{\alpha}\right) d s \\
& \mathbf{k}_{\Omega w}^{\sigma s}=\int_{0}^{b_{e l}}\left(t_{e l} \nu E_{s} \mathbf{N}_{\Omega}^{\top} \mathbf{N}_{s, s}-\frac{t_{e l}^{3}}{12} \nu E_{s} \mathbf{N}_{\alpha}^{\top} \mathbf{N}_{n, s s}\right) d s \\
& \mathbf{k}_{\Omega \Omega}^{\gamma}=\int_{0}^{b_{e l}}\left(t_{e l} G \mathbf{N}_{\alpha}^{\top} \mathbf{N}_{\alpha}+t_{e l} G \mathbf{N}_{\Omega, s}^{\top} \mathbf{N}_{\Omega, s}+\frac{t_{e l}^{3}}{12} G \mathbf{N}_{\alpha, s}^{\top} \mathbf{N}_{\alpha, s}\right) d s \\
& \mathbf{k}_{w w}^{\gamma}=\int_{0}^{b_{e l}}\left(t_{e l} G \mathbf{N}_{n}^{\top} \mathbf{N}_{n}+t_{e l} G \mathbf{N}_{s}^{\top} \mathbf{N}_{s}+\frac{t_{e l}^{3}}{12} G \mathbf{N}_{n, s}^{\top} \mathbf{N}_{n, s}\right) d s \\
& \mathbf{k}_{\Omega w}^{\gamma}=\int_{0}^{b_{e l}}\left(t_{e l} G \mathbf{N}_{\alpha}^{\top} \mathbf{N}_{n}+t_{e l} G \mathbf{N}_{\Omega, s}^{\top} \mathbf{N}_{s}-\frac{t_{e l}^{3}}{12} G \mathbf{N}_{\alpha, s}^{\top} \mathbf{N}_{n, s}\right) d s \\
& \mathbf{k}_{w \Omega}^{\gamma}=\int_{0}^{b_{e l}}\left(t_{e l} G \mathbf{N}_{n}^{\top} \mathbf{N}_{\alpha}+t_{e l} G \mathbf{N}_{s}^{\top} \mathbf{N}_{\Omega, s}-\frac{t_{e l}^{3}}{12} G \mathbf{N}_{n, s}^{\top} \mathbf{N}_{\alpha, s}\right) d s \\
&
\end{aligned}
$$

Formulating the assembled cross section stiffness matrices and vectors containing all degrees of freedom it is convenient to express the local degrees in terms of a global reference frame. Thus, using a formal standard transformation matrix, the local degrees are written in global coordinates $\left(\mathbf{v}^{e l}=\mathbf{T}^{e l} \mathbf{v}\right)$. Therefore, the local stiffness matrices can be assembled using a common assembling procedure yielding global cross sectional stiffness matrices (see e.g. [20]). Following this procedure, the strain energy from equation (7) takes the form:

$$
U=\frac{1}{2} \int_{0}^{\ell}\left[\begin{array}{c}
\mathbf{v}_{w} \psi \\
\mathbf{v}_{\Omega} \eta \\
\mathbf{v}_{w} \psi^{\prime} \\
\mathbf{v}_{\Omega} \eta^{\prime}
\end{array}\right]^{\top}\left[\begin{array}{cccc}
\mathbf{K}_{w w}^{s} & \cdot & \cdot & \mathbf{K}_{w \Omega}^{s \sigma} \\
\cdot & \mathbf{K}_{\Omega \Omega}^{\gamma} & \mathbf{K}_{\Omega w}^{\gamma} & \cdot \\
\cdot & \mathbf{K}_{w \Omega}^{\gamma} & \mathbf{K}_{w w}^{\gamma} & \cdot \\
\mathbf{K}_{\Omega w}^{\sigma s} & \cdot & \cdot & \mathbf{K}_{\Omega \Omega}^{\sigma}
\end{array}\right]\left[\begin{array}{c}
\mathbf{v}_{w} \psi \\
\mathbf{v}_{\Omega} \eta \\
\mathbf{v}_{w} \psi^{\prime} \\
\mathbf{v}_{\Omega} \eta^{\prime}
\end{array}\right] d z
$$

using global stiffness matrices. Here and in the following a dot '.' represents a suitable size null-matrix.

\subsection{Displacement mode equilibrium}

The differential equations for the displacement modes are derived using variational principles on the strain en- 
ergy functional. The delta symbols, $\delta$, in front of the displacement vectors are introduced to represent the variational terms. Integration by parts is performed on the variational terms, which are differentiated with respect to the axial coordinate. Thereby we eliminate axial derivatives (the primes) of the variational terms $\delta\left(\mathbf{v}_{w} \psi^{\prime}\right)$ and $\delta\left(\mathbf{v}_{\Omega} \eta^{\prime}\right)$ and the first variation of the strain energy takes the form:

$$
\begin{array}{r}
\delta U=\int_{0}^{\ell} \delta\left[\begin{array}{l}
\mathbf{v}_{w} \psi \\
\mathbf{v}_{\Omega} \eta
\end{array}\right]^{\top}\left(\left[\begin{array}{cc}
-\mathbf{K}_{w w}^{\gamma} & \cdot \\
\cdot & -\mathbf{K}_{\Omega \Omega}^{\sigma}
\end{array}\right]\left[\begin{array}{l}
\mathbf{v}_{w} \psi \\
\mathbf{v}_{\Omega} \eta
\end{array}\right]^{\prime \prime}\right. \\
+\left[\begin{array}{cc}
\cdot & \mathbf{K}_{w \Omega}^{s \sigma}-\mathbf{K}_{w \Omega}^{\gamma} \\
\mathbf{K}_{\Omega w}^{\gamma}-\mathbf{K}_{\Omega w}^{\sigma s} & \cdot
\end{array}\right]\left[\begin{array}{l}
\mathbf{v}_{w} \psi \\
\mathbf{v}_{\Omega} \eta
\end{array}\right]^{\prime} \\
\left.+\left[\begin{array}{cc}
\mathbf{K}_{w w}^{s} & \cdot \\
\cdot & \mathbf{K}_{\Omega \Omega}^{\gamma}
\end{array}\right]\left[\begin{array}{c}
\mathbf{v}_{w} \psi \\
\mathbf{v}_{\Omega} \eta
\end{array}\right]\right) d z \\
+\left[\delta [ \begin{array} { l l } 
{ \mathbf { v } _ { w } \psi } \\
{ \mathbf { v } _ { \Omega } \eta }
\end{array} ] ^ { \top } \left(\left[\begin{array}{cc}
\mathbf{K}_{w w}^{\gamma} & \cdot \\
\cdot & \mathbf{K}_{\Omega \Omega}^{\sigma}
\end{array}\right]\left[\begin{array}{l}
\mathbf{v}_{w} \psi \\
\mathbf{v}_{\Omega} \eta
\end{array}\right]^{\prime}\right.\right. \\
\left.\left.+\left[\begin{array}{cc}
\cdot & \mathbf{K}_{w \Omega}^{\gamma} \\
\mathbf{K}_{\Omega w}^{\sigma s} & \cdot
\end{array}\right]\left[\begin{array}{l}
\mathbf{v}_{w} \psi \\
\mathbf{v}_{\Omega} \eta
\end{array}\right]\right)\right]_{0}^{\ell}
\end{array}
$$

In this equation the last bracketed terms, i.e. $[\cdots]_{0}^{\ell}$, represent boundary terms, that ensure equilibrium with the boundary loads, which we have not introduced at this stage.

It follows from calculus of variations that, the first variation of the energy must vanish in order to have minimum energy. Therefore, the $\delta U$ should vanish, i.e. $\delta U=0$, for arbitrary variations in $\delta\left(\mathbf{v}_{w} \psi\right)$ and $\delta\left(\mathbf{v}_{\Omega} \eta\right)$, satisfying kinematic boundary conditions. This requirement is the condition of stationarity, which leads to the following set of equilibrium equations.

$$
\begin{gathered}
\underbrace{\left[\begin{array}{cc}
-\mathbf{K}_{w w}^{\gamma} & \cdot \\
\cdot & \mathbf{K}_{\Omega \Omega}^{\sigma}
\end{array}\right]}_{\mathbf{K}_{2}} \underbrace{\left[\begin{array}{c}
\mathbf{v}_{w} \psi \\
\mathbf{v}_{\Omega} \eta
\end{array}\right]}_{\mathbf{u}^{\prime \prime}} \\
+\underbrace{\left[\begin{array}{cc}
\mathbf{K}_{\Omega w}^{\sigma s}-\mathbf{K}_{\Omega w}^{\gamma} & \mathbf{K}_{w \Omega}^{s \sigma}-\mathbf{K}_{w \Omega}^{\gamma} \\
\cdot
\end{array}\right]}_{\mathbf{K}_{1}} \underbrace{\left[\begin{array}{l}
\mathbf{v}_{w} \psi \\
\mathbf{v}_{\Omega} \eta
\end{array}\right]^{\prime}}_{\mathbf{u}^{\prime}} \\
+\underbrace{\left[\begin{array}{cc}
\mathbf{K}_{w w}^{s} \cdot \\
\cdot & -\mathbf{K}_{\Omega \Omega}^{\gamma}
\end{array}\right]}_{\mathbf{K}_{0}} \underbrace{\left[\begin{array}{l}
\mathbf{v}_{w} \psi \\
\mathbf{v}_{\Omega} \eta
\end{array}\right]}_{\mathbf{u}}=\left[\begin{array}{c}
\mathbf{0} \\
\mathbf{0}
\end{array}\right]
\end{gathered}
$$

Please note that to achieve symmetry in the matrix formulation the lower block-row-equation is multiplied by -1 . Furthermore, we have, in the above equation, introduced the matrices $\mathbf{K}_{2}, \mathbf{K}_{1}$ and $\mathbf{K}_{0}$ as well as the vector of displacements $\mathbf{u}$. This has been done in order to obtain the following more compact formulation of these differential equilibrium equations:

$$
\mathbf{K}_{2} \mathbf{u}^{\prime \prime}+\mathbf{K}_{1} \mathbf{u}^{\prime}+\mathbf{K}_{0} \mathbf{u}=\mathbf{0}
$$

Consequently, we have achieved a set of second order displacement mode differential equations and not as in other higher order beam theories, such as GBT, a fourth order differential equation system. The reason why a secondorder beam differential equation system is derived here, is the use of warping fields $\Omega \eta$ and $\alpha \eta$, which until otherwise stated are independent of $\psi$.

\section{Cross section mode determination procedure}

The cross section mode determination procedure reflects the solution of the displacement mode differential equations using the approach of assuming exponential solutions and finding these through the solution of the characteristic equations being a quadratic eigenvalue problem. However, hereby we have not found the complete homogeneous solution, since there will be $n_{z}=12$ null eigenvalues corresponding to the classic polynomial solutions including constant strain modes and rigid body motions also referred to as fundamental modes. The polynomial solutions are found by seeking a set of $n_{z}$ third order polynomial solutions. Since there are a multitude of possible combinations of these modes an engineering based procedure, which enables the direct relation to classic beam theory, is described. This task, however, is not as straight forward as expected.

\subsection{Exponential modes}

Let us start out seeking exponential solutions. In the previous section separation of variables was used to formulate displacements as product terms of cross sectional displacements and the amplitude functions. Thus, we need to find solutions to the differential equations $(13)$ with the displacement vector:

$$
\mathbf{u}(z)=\left[\begin{array}{l}
\mathbf{v}_{w} \psi(z) \\
\mathbf{v}_{\Omega} \eta(z)
\end{array}\right]
$$

in which the vectors $\mathbf{v}_{w}$ and $\mathbf{v}_{\Omega}$ contains cross sectional degrees of freedom with respect to transverse and warping displacements, respectively. It is clear that two related amplitude functions $\psi(z)$ and $\eta(z)$ have been introduced. This, however, has been done in order to keep the relation to conventional formulations of beam theories. Nonetheless, to solve the coupled differential equations we now assume that $\eta=\psi$. This enables us to write the displacement using a single amplitude function as:

$$
\mathbf{u}(z)=\mathbf{v} \psi(z) c
$$

in which the cross section displacement mode vector is given by:

$$
\mathbf{v}=\left[\begin{array}{l}
\mathbf{v}_{w} \\
\mathbf{v}_{\Omega}
\end{array}\right]
$$


The complex constant to be determined by boundary conditions, $c_{p} \in \mathbb{C}$ has been introduced in equation 15 to scale the whole solution mode.

A general solution of the beam differential equations 13 is sought by assuming an exponential amplitude function: $\psi=e^{\lambda z}$, whereby the assumed solution vector from equation 15 becomes:

$$
\mathbf{u}_{e}=\mathbf{v}_{e} e^{\lambda z} c_{e}
$$

Here, we have introduced the subscript $e$ to distinguish an exponential solution mode from a polynomial one. Inserting this assumed solution into the differential equations 13 and simplifying by dividing by $e^{\lambda z} c_{e}$ the following algebraic system of equations is found.

$$
\left(\lambda^{2} \mathbf{K}_{2}+\lambda \mathbf{K}_{1}+\mathbf{K}_{0}\right) \mathbf{v}_{e}=\mathbf{0} \text { with } \lambda \in \mathbb{C}
$$

This algebraic system of equations is a quadratic matrix eigenvalue problem with $\lambda$ as the eigenvalue variable and $\mathbf{v}_{e}$ being the eigenvector representing an associated deformation mode. Solution modes can be derived by reducing the differential order of equation $(13)$ to an equivalent system of first order differential equations introducing a state vector as proposed by Tisseur and Meerbergen [21] leading to a generalized eigenvalue problem but also doubling the number of solution modes, or directly by solving the quadratic eigenvalue problem by use of the built-in function polyeig in MATLAB [22. It is worth noting that it is the state vectors that become orthogonal (with respect to the total strain energy).

The eigenvectors associated with non-null eigenvalues are the cross section displacement vectors related to the exponential modes. The eigenvalues can be viewed as inverse length scale parameters and the importance of each mode depends on the length scale. The larger the scale, the more important is the related displacement mode in relation to the beam model. Thus, we will sort the modes hierarchically such that modes with low eigenvalues are those of higher importance and comes first. The more localized and detailed behaviour that has to be assessed by the beam model, the more short scale modes will have to be included. The sum of all exponential modes may be written as:

$$
\mathbf{u}_{e}=\mathbf{V}_{e} \boldsymbol{\Psi}_{e} \mathbf{c}_{e}
$$

where $\mathbf{V}_{e}$ contains all the sorted eigenvectors of the associated non-null eigenvalues $\lambda_{i}$ as its columns, $\boldsymbol{\Psi}_{e}$ is a diagonal amplitude matrix and $\mathbf{c}_{e}$ is a column vector containing a constant to each mode. In other words, the components in equation 19 are given as follows:

$$
\begin{gathered}
\mathbf{V}_{e}=\left[\begin{array}{llll}
\mathbf{v}_{e_{1}} & \mathbf{v}_{e_{2}} & \ldots & \mathbf{v}_{e_{n_{e}}}
\end{array}\right] \\
\mathbf{\Psi}_{e}=\left[\begin{array}{cccc}
\psi_{e_{1}} & & & \\
& \psi_{e_{2}} & & \\
& & \ddots & \\
& & & \psi_{e_{n_{e}}}
\end{array}\right], \quad \mathbf{c}_{e}=\left[\begin{array}{c}
c_{e_{1}} \\
c_{e_{2}} \\
\vdots \\
c_{e_{n_{e}}}
\end{array}\right]
\end{gathered}
$$

in which the $i$ th exponential diagonal amplitude function is given as $\psi_{e_{i}}=e^{\lambda_{i} z}$ and where $n_{e}$ is the number of exponential modes being $n_{e}=2 n-n_{z}$, where $n$ is six times the number of nodes in the discretized cross section.

Not considering the null-eigenvalues the solution of the quadratic eigenvalue problem contains pairs of real eigenvalues $\{\lambda,-\lambda\}$ as well as complex quadruples $\{\lambda,-\lambda, \bar{\lambda},-\bar{\lambda}\}$. The quadruples have a real part representing the axial decay effect accompanied by an oscillatory amplitude related to the imaginary part. In case of complex eigenvalues the eigenvectors also become complex.

To be able to present and discuss unique eigenmodes, all the distortional vectors in $\mathbf{V}_{e}$ have been normalized by multiplication by a complex constant. This constant is determined in such a way that the largest absolute value of all components in each transverse displacement vector $\mathbf{v}_{w}$ is one and that this component is real. This has to be done in order to make them unique since complex eigenvectors are determined by the eigenvalue problem except for an arbitrary complex constant given in $\mathbf{c}_{e}$.

The eigenvectors related to the $n_{z}=12$ null-eigenvalues are coupled, since many of them are related to rigid body displacements and constant strain displacement fields, which have polynomial amplitude functions. The following subsection derives these fundamental modes.

\subsection{Fundamental modes}

Eigenvectors associated with null-eigenvalues of the quadratic eigenvalue problem in equation (18) are assumed to be fundamental beam modes having a polynomial amplitude function along the beam axis without any exponential components. These represent the classical beam mode solutions as those illustrated in figure 4. From classic beam theory, we know that the rigid body displacements corresponds to constant and linear amplitude functions, that pure extension and unrestrained torsion corresponds to constant strain and linear displacements, where as constant bending strain corresponds to quadratic displacements. Moreover, constant shear strain does not exist alone but corresponds to a mode that also has linear varying bending strains and therefore has a cubic variation of the transverse displacements. It is important to note that the deformation shapes are not known beforehand as in ordinary beam theory, since shear deformation and the Poisson expansion of the cross section are now included in the theory. With this in mind, we assume a third order polynomial function to embrace the fundamental modes. Thus, let us seek $n_{z}$ solutions of the form:

$$
\mathbf{u}_{p}=\left(\frac{z^{3}}{3 !} \mathbf{v}_{3}+\frac{z^{2}}{2 !} \mathbf{v}_{2}+z \mathbf{v}_{1}+\mathbf{v}_{0}\right) c_{p}
$$

where $\left\{\mathbf{v}_{3}, \mathbf{v}_{2}, \mathbf{v}_{1}, \mathbf{v}_{0}\right\}$ is a set of cross section deformation vectors altogether representing one polynomial displacement mode factored by the constant $c_{p}$. Each vector contains both the in-plane and out-of-plan components as 
in equation (16). Now, substituting this assumed polynomial solution function and its derivatives into the beam differential equations from equation $(13)$ results in the following set of equations that must be fulfilled in order to maintain equilibrium:

$$
\begin{aligned}
& \left(\frac{1}{6} z^{3}\left(\mathbf{K}_{0} \mathbf{v}_{3}\right)+\frac{1}{2} z^{2}\left(\mathbf{K}_{1} \mathbf{v}_{3}+\mathbf{K}_{0} \mathbf{v}_{2}\right)\right. \\
& +z\left(\mathbf{K}_{2} \mathbf{v}_{3}+\mathbf{K}_{1} \mathbf{v}_{2}+\mathbf{K}_{0} \mathbf{v}_{1}\right) \\
& \left.\quad+\left(\mathbf{K}_{2} \mathbf{v}_{2}+\mathbf{K}_{1} \mathbf{v}_{1}+\mathbf{K}_{0} \mathbf{v}_{0}\right)\right) c_{p}=0
\end{aligned}
$$

By assembling the polynomial terms in a vector and assembling the deformation mode vectors in a vector as well, the equilibrium equations may be expressed by an assembled matrix notation as follows:

$$
\left[\begin{array}{r}
\frac{1}{6} z^{3} \mathbf{I}_{n} \\
\frac{1}{2} z^{2} \mathbf{I}_{n} \\
z \mathbf{I}_{n} \\
\mathbf{I}_{n}
\end{array}\right]^{\top}\left[\begin{array}{cccc}
\mathbf{K}_{0} & \cdot & \cdot & \cdot \\
\mathbf{K}_{1} & \mathbf{K}_{0} & \cdot & \cdot \\
\mathbf{K}_{2} & \mathbf{K}_{1} & \mathbf{K}_{0} & \cdot \\
\cdot & \mathbf{K}_{2} & \mathbf{K}_{1} & \mathbf{K}_{0}
\end{array}\right]\left[\begin{array}{c}
\mathbf{v}_{3} \\
\mathbf{v}_{2} \\
\mathbf{v}_{1} \\
\mathbf{v}_{0}
\end{array}\right] c_{p}=\mathbf{0}
$$

In which we have introduced the unit matrix $\mathbf{I}_{n}$, where $n$ is a reference to the number of degrees of freedom in each block.

\section{Mode space of the fundamental modes}

The non-trivial solutions to these equilibrium equations 22 , i.e. with $c_{p} \neq 0$ for all values of $z$, can be found as the null space of $\mathbb{K}$ being:

$$
\mathbb{V}=\mathcal{N}(\mathbb{K})
$$

where $\mathcal{N}$ is the null space algebraic operation and the solution satisfies $\mathbb{K} \mathbb{V}=\mathbf{0}$ where $\mathbb{K}$ is given in equation 25 by the block matrices defined in equation $\sqrt{12}$ and $\mathbb{V}$ may be separated into equivalent blocks also illustrated in equation (25). The subscripts on the block matrices $\mathbf{V}_{k}$ in $\mathbb{V}$ refers to their position in the polynomial solution from equation (21) - the coefficient numbers $k$ reflects the associated polynomial order of $z$, i.e. a column in $\mathbf{V}_{k}$ will be linked to $\frac{1}{k !} z^{k}$.

$$
\mathbb{K}=\left[\begin{array}{cccc}
\mathbf{K}_{0} & \cdot & \cdot & \cdot \\
\mathbf{K}_{1} & \mathbf{K}_{0} & \cdot & \cdot \\
\mathbf{K}_{2} & \mathbf{K}_{1} & \mathbf{K}_{0} & \cdot \\
\cdot & \mathbf{K}_{2} & \mathbf{K}_{1} & \mathbf{K}_{0}
\end{array}\right] \text { and } \mathbb{V}=\left[\begin{array}{c}
\mathbf{V}_{3} \\
\mathbf{V}_{2} \\
\mathbf{V}_{1} \\
\mathbf{V}_{0}
\end{array}\right]
$$

Computing the null space, in equation (24), yields twelve solutions given as the columns in $\mathbb{V}$. This corresponds exactly to the number of null-eigenvalues associated with the quadratic eigenvalue problem in equation (18). Moreover, this also corresponds to the number of classic solutions, e.g. the elementary solutions considered by Morandini et al. 15] determined using a slightly different procedure considering a Jordan chain formulation. However, the modes found as solutions to the null space of $\mathbb{K}$ are coupled in the sense that they represent a mix of the classic beam modes. These modes may be used in the continued beam analysis, however, they would not give a clear engineering connection to classic beam theory. Hence, a mode identification procedure is required in order to decouple classic solution modes, which are representative.

From an engineering perspective, we know that the twelve fundamental solution modes can be subdivided into six rigid body deformation modes; and six strain modes corresponding to pure extension, (unconstrained) free torsion, pure bending and constant shear strain with linear varying normal strains; as illustrated in figure 4 From the displacement fields, illustrated in the figure, it can be deduced that the individual solution modes may have contributions from up to four cross section displacement vectors $\mathbf{v}_{3}, \mathbf{v}_{2}, \mathbf{v}_{1}$ and $\mathbf{v}_{0}$ with the corresponding polynomial amplitude functions $\left\{\frac{1}{6} z^{3}, \frac{1}{2} z^{2}, z, 1\right\}$ according to equation (21). Note that each of these four cross section displacement vectors $\mathbf{v}_{k}$ have a transverse part $\mathbf{v}_{k w}$ and a warping part $\mathbf{v}_{k \Omega}$ as indicated in equation (16). Table 3 gives an overview of the twelve fundamental modes in relation to cross section displacement vectors and their related polynomial terms as seen from an engineering point of view. The intent of the fourth line in the table is to give a reference to the principal axis of displacement, rotation or bending as 1 or 2 diThe fundamental modes shown in figure 13 and 14 of the results section 4 may clarify the principle. However, the displacement vectors need to be found and identified. Furthermore, from table 3 it can be seen that this subdivision of fundamental modes leads to a logical separation based on polynomial order related to these modes. Thus, the polynomial order may be used in a mode identification procedure, which follows in next section.

\section{Identification of modes dependent on polynomial order}

The procedure adopted to identify the cubic, quadratic, linear and constant amplitude function modes is similar to the procedure described by Vieira [23. The ordering and the orthogonalization procedure, which we apply is new. The orthogonalization procedure is based on strain energy for strain modes and on inertial energy for rigid modes.

In the following, four superscripts are introduced - one for each polynomial "family". The four superscripts used for sub-columns of $\mathbb{V}$ are: $\mathrm{C}, \mathrm{L}, \mathrm{B}$ and $\mathrm{S}$ assigned to constant modes of zero order, linear modes of first order, bending modes of second order, and shear modes of third order, respectively. Having found the null space in equation (24), the columns in the block vector $\mathbf{V}_{3}$ contains the displacement content of third order. From our engineering viewpoint only two modes with constant shear and linear bending should be identified with this particular order. Therefore the columns in $\mathbb{V}$ must be decoupled using $\mathbf{V}_{3}$. To do this and in order to identify displacement modes with a cubic content, a singular value decomposition (SVD) is applied to $\mathbf{V}_{3}$. The SVD results in a decomposition of $\mathbf{V}_{3}$ in a null part "." and a non-null part $\mathbf{V}_{3}^{\mathrm{S}}$. However, the 

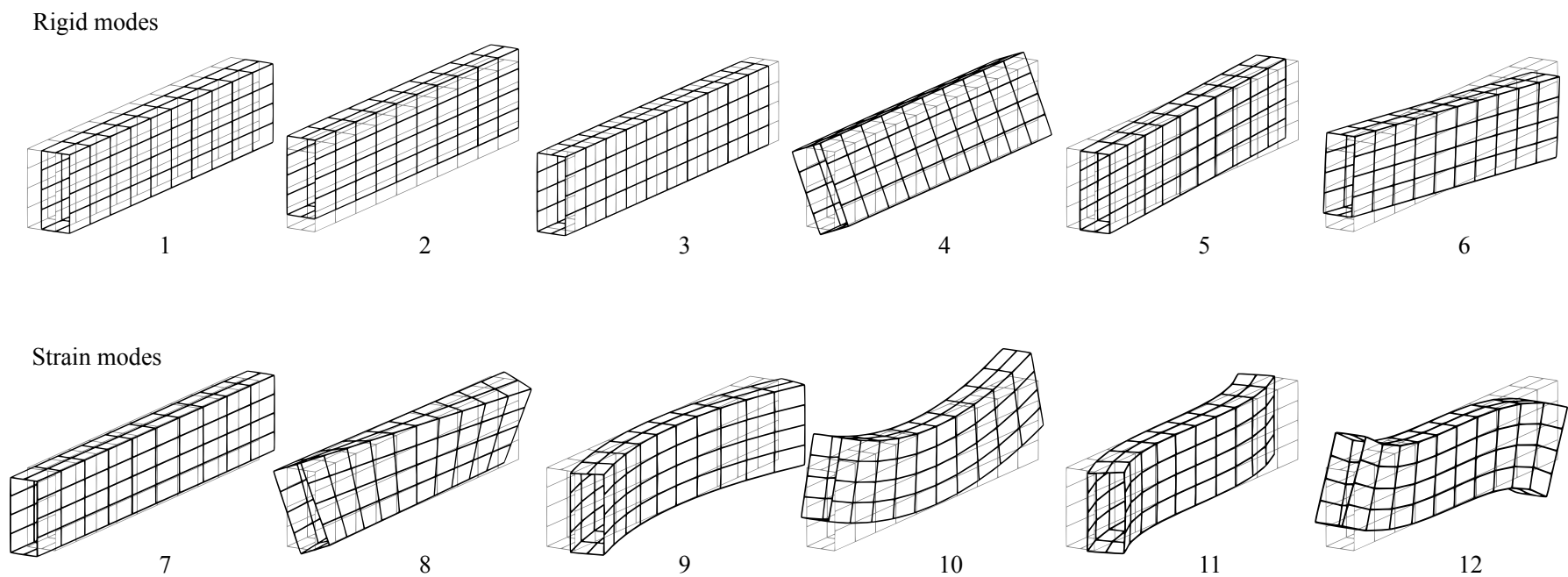

Figure 4: Illustration of fundamental displacement modes

Table 3: Amplitude functions of cross section displacement vectors related to the fundamental displacement modes.

\begin{tabular}{|c|c|c|c|c|c|c|c|c|c|c|c|c|c|}
\hline \multirow{3}{*}{\multicolumn{2}{|c|}{ Type }} & \multicolumn{6}{|c|}{ Rigid modes } & \multicolumn{6}{|c|}{ Strain modes } \\
\hline & & \multicolumn{3}{|c|}{$\begin{array}{c}\text { Translations } \\
\text { in }\end{array}$} & \multicolumn{3}{|c|}{$\begin{array}{c}\text { Rotations } \\
\text { about }\end{array}$} & \multicolumn{2}{|c|}{$\begin{array}{l}\text { Extension } \\
\text { \& Torsion }\end{array}$} & \multicolumn{2}{|c|}{$\begin{array}{c}\text { Pure } \\
\text { Bending abt }\end{array}$} & \multicolumn{2}{|c|}{$\begin{array}{l}\text { Constant } \\
\text { Shear in }\end{array}$} \\
\hline & & $X$ & $Y$ & $Z$ & $Z$ & $Y$ & $X$ & Ext. & Tors. & Y & $\mathrm{X}$ & $\mathrm{X}$ & $\mathrm{Y}$ \\
\hline \multicolumn{2}{|c|}{ Mode $i$} & 1 & 2 & 3 & 4 & 5 & 6 & 7 & 8 & 9 & 10 & 11 & 12 \\
\hline $\mathbf{V}_{3}$ & $\begin{array}{l}\mathbf{v}_{3 w}^{i} \\
\mathbf{v}_{3 \Omega}^{i}\end{array}$ & & & & & & & & & & & $\frac{1}{6} z^{3}$ & $\frac{1}{6} z^{3}$ \\
\hline $\mathbf{V}_{2}$ & $\begin{array}{l}\mathbf{v}_{2 w}^{i} \\
\mathbf{v}_{2 \Omega}^{i}\end{array}$ & & & & & & & & & $\frac{1}{2} z^{2}$ & $\frac{1}{2} z^{2}$ & $\frac{1}{2} z^{2}$ & $\frac{1}{2} z^{2}$ \\
\hline $\mathbf{V}_{1}$ & $\begin{array}{l}\mathbf{v}_{1 w}^{i} \\
\mathbf{v}_{1 \Omega}^{i}\end{array}$ & & & & & $z$ & $z$ & $z$ & $z$ & $z$ & $z$ & $z^{\dagger}$ & $z^{\dagger}$ \\
\hline $\mathbf{V}_{0}$ & $\begin{array}{l}\mathbf{v}_{0 w}^{i} \\
\mathbf{v}_{0 \Omega}^{i}\end{array}$ & 1 & 1 & 1 & 1 & 1 & 1 & $1^{\dagger}$ & 1 & $1^{\dagger}$ & $1^{\dagger}$ & 1 & 1 \\
\hline \multicolumn{2}{|c|}{ Max. order } & & & & \multicolumn{2}{|c|}{1} & \multicolumn{2}{|c|}{2} & \\
\hline
\end{tabular}

$\nmid$ Poisson effect 
decomposition is applied not only to $\mathbf{V}_{3}$ but to the whole mode space matrix $\mathbb{V}$, such that it is subdivided into a remaining mode space $\mathbf{V}^{r}$ of lower order and a third order mode space $\mathbf{V}^{\mathrm{S}}$, which we relate to shear. Hence, the null space of $\mathbb{K}$ takes the form $\mathbb{V}=\left[\mathbf{V}^{r}, \mathbf{V}^{\mathrm{S}}\right]$, which may also be written as:

$$
\mathbb{V}=\left[\begin{array}{cc}
\cdot & \mathbf{V}_{3}^{\mathrm{S}} \\
\mathbf{V}_{2}^{r} & \mathbf{V}_{2}^{\mathrm{S}} \\
\mathbf{V}_{1}^{r} & \mathbf{V}_{1}^{\mathrm{S}} \\
\mathbf{V}_{0}^{r} & \mathbf{V}_{0}^{\mathrm{S}}
\end{array}\right]
$$

Next, the modes of second order, i.e. bending modes, are identified by performing a SVD on the remaining block matrix $\mathbf{V}_{2}^{r}$. This similarly leads to a decoupling into new remaining modes $\mathbf{V}^{n r}$ and second order bending modes $\mathbf{V}^{\mathrm{B}}$. The null space of $\mathbb{K}$ now has the form $\mathbb{V}=\left[\mathbf{V}^{n r}, \mathbf{V}^{\mathrm{B}}, \mathbf{V}^{\mathrm{S}}\right]$. Finally, the last step is to decouple linear and constant terms through a SVD on the remaining sub-matrix $\mathbf{V}_{1}^{n r}$, whereafter those modes related to the non-null singular values receive a superscript $\mathrm{L}$ where as the remaining part a superscript $\mathrm{C}$. The number of modes having a given order complies with the numbers expected from table 3 Furthermore, the result of this successive procedure is that we have decomposed the polynomial displacement modes according to polynomial order and the matrix $\mathbb{V}$ now takes the form:

$$
\mathbb{V}=\left[\begin{array}{cccc}
\cdot & \cdot & \cdot & \mathbf{V}_{3}^{\mathrm{S}} \\
\cdot & \cdot & \mathbf{V}_{2}^{\mathrm{B}} & \mathbf{V}_{2}^{\mathrm{S}} \\
\cdot & \mathbf{V}_{1}^{\mathrm{L}} & \mathbf{V}_{1}^{\mathrm{B}} & \mathbf{V}_{1}^{\mathrm{S}} \\
\mathbf{V}_{0}^{\mathrm{C}} & \mathbf{V}_{0}^{\mathrm{L}} & \mathbf{V}_{0}^{\mathrm{B}} & \mathbf{V}_{0}^{\mathrm{S}}
\end{array}\right]
$$

Even though the modes have been identified and sorted according to their polynomial order, the linear modes are not separated into a rigid part and a strain part. Furthermore, the modes are not pure and for example the third order modes $\mathbf{V}^{\mathrm{S}}$ may be contaminated by parts of the lower order modes $\mathbf{V}^{\mathrm{B}}, \mathbf{V}^{\mathrm{L}}$ and $\mathbf{V}^{\mathrm{C}}$ and so forth. It would also be convenient that the strain modes are orthogonal with respect to strain energy, for example that they represents the principal axis bending modes.

To achieve all this, a procedure following the steps listed here, will be executed on the modes in equation (27).

a) Split linear modes into rigid modes and orthogonal strain modes.

b) Remove lower order strain modes from higher order strain modes.

c) Find principal bending modes from the second order modes.

d) Find shear modes following the principal axis of bending.

e) Transform rigid modes according to principal directions. f) Remove lower order rigid modes from higher order modes.

The following subsections will describe the individual procedural steps.

\section{a) Split linear modes into rigid modes and strain modes}

To identify the classic beam modes we need to introduce and relate to the strain energy formulated with respect to the polynomial cross section displacement modes. In this regard, consider the strain energy from equation (10), where the integration over the beam length is not of interest since we investigate cross section behaviours at an arbitrary point along the beam, however, the energy depends on the product of the amplitude functions. Thus, we consider the strain energy in a cross section or rather the strain energy density per beam length, which takes the form:

$$
U^{\prime}=\frac{1}{2}\left[\begin{array}{c}
\mathbf{v}_{w} \psi \\
\mathbf{v}_{\Omega} \eta \\
\mathbf{v}_{w} \psi^{\prime} \\
\mathbf{v}_{\Omega} \eta^{\prime}
\end{array}\right]^{\top}\left[\begin{array}{cccc}
\mathbf{K}_{w w}^{s} & \cdot & \cdot & \mathbf{K}_{w \Omega}^{s \sigma} \\
\cdot & \mathbf{K}_{\Omega \Omega}^{\gamma} & \mathbf{K}_{\Omega w}^{\gamma} & \cdot \\
\cdot & \mathbf{K}_{w \Omega}^{\gamma} & \mathbf{K}_{w w}^{\gamma} & \cdot \\
\mathbf{K}_{\Omega w}^{\sigma s} & \cdot & \cdot & \mathbf{K}_{\Omega \Omega}^{\sigma}
\end{array}\right]\left[\begin{array}{c}
\mathbf{v}_{w} \psi \\
\mathbf{v}_{\Omega} \eta \\
\mathbf{v}_{w} \psi^{\prime} \\
\mathbf{v}_{\Omega} \eta^{\prime}
\end{array}\right]
$$

As stated previously, we assume the functions $\psi$ and $\eta$ to be polynomials of third order multiplied by a set of coefficient vectors introduced as displacement vectors. As a result, the mode vectors in equation (28) may be formulated, for a single mode, as:

$$
\begin{aligned}
& \mathbf{v}_{w} \psi=\frac{1}{6} \mathbf{v}_{3 w} z^{3}+\frac{1}{2} \mathbf{v}_{2 w} z^{2}+\mathbf{v}_{1 w} z+\mathbf{v}_{0 w} \\
& \mathbf{v}_{\Omega} \eta=\frac{1}{6} \mathbf{v}_{3 \Omega} z^{3}+\frac{1}{2} \mathbf{v}_{2 \Omega} z^{2}+\mathbf{v}_{1 \Omega} z+\mathbf{v}_{0 \Omega}
\end{aligned}
$$

and its first derivatives as:

$$
\begin{aligned}
& \mathbf{v}_{w} \psi^{\prime}=\frac{1}{2} \mathbf{v}_{3 w} z^{2}+\mathbf{v}_{2 w} z+\mathbf{v}_{1 w} \\
& \mathbf{v}_{\Omega} \eta^{\prime}=\frac{1}{2} \mathbf{v}_{3 \Omega} z^{2}+\mathbf{v}_{2 \Omega} z+\mathbf{v}_{1 \Omega}
\end{aligned}
$$

Based on our engineering intuition and mathematics we foresee the following:

- Inserting the zero order rigid modes $\mathbf{V}^{\mathrm{C}}$ will all result in zero energy density, hence, all being rigid motions.

- For the first order modes $\mathbf{V}^{\mathrm{L}}$ corresponding to rotational rigid body modes, extension and torsion will result in four modes with some constant energy density, since the modes are not pure.

- The second order modes $\mathbf{V}^{\mathrm{B}}$ corresponding to bending will result in two modes with constant energy density.

- The third order modes $\mathbf{V}^{\mathrm{S}}$ will result in a constant energy density term corresponding to pure shear and a quadratic energy term corresponding to linear varying bending moments.

Consequently, it is convenient to introduce an inner product based on the strain energy density to be able to com- 
bine mode vectors. This inner product we formulate as:

$$
\begin{aligned}
& \left\langle\left[\begin{array}{c}
\mathbf{V}_{k}^{i} \\
\mathbf{V}_{(k+1)}^{i}
\end{array}\right],\left[\begin{array}{c}
\mathbf{V}_{l}^{j} \\
\mathbf{V}_{(l+1)}^{j}
\end{array}\right]\right\rangle_{E} \\
& =\left[\begin{array}{c}
\mathbf{v}_{k w}^{i} \\
\mathbf{v}_{k \Omega}^{i} \\
\mathbf{v}_{(k+1) w}^{i} \\
\mathbf{v}_{(k+1) \Omega}^{i}
\end{array}\right]^{\top}\left[\begin{array}{cccc}
\mathbf{K}_{w w}^{s} & \cdot & \cdot & \mathbf{K}_{w \Omega}^{s \sigma} \\
\cdot & \mathbf{K}_{\Omega \Omega}^{\gamma} & \mathbf{K}_{\Omega w}^{\gamma} & \cdot \\
\cdot & \mathbf{K}_{w \Omega}^{\gamma} & \mathbf{K}_{w w}^{\gamma} & \cdot \\
\mathbf{K}_{\Omega w}^{\sigma s} & \cdot & \cdot & \mathbf{K}_{\Omega \Omega}^{\sigma}
\end{array}\right]\left[\begin{array}{c}
\mathbf{v}_{l w}^{j} \\
\mathbf{v}_{l \Omega}^{j} \\
\mathbf{v}_{(l+1) w}^{j} \\
\mathbf{v}_{(l+1) \Omega}^{j}
\end{array}\right]
\end{aligned}
$$

If $\mathbf{V}^{i}$ and $\mathbf{V}^{j}$ represent columns $i$ and $j$, the inner product computes a number (representing the work and related stiffness of the two modes). On the other hand if $\mathbf{V}^{i}$ and $\mathbf{V}^{j}$ each represent a number of columns (for example a mode family) the inner product computes a subspace (stiffness) matrix related to these modes. Thus, the subscripts $k$ and $l$ refer to the coefficient numbers related to the polynomial degree (see equation (25)) whereas $i$ and $j$ refers to the solution family or a column number. Note that the +1 in subscripts $(k+1)$ and $(l+1)$ stem from differentiated terms in equation (30); when for example gathering energy terms of constant or quadratic dependency on $z$.

In order to separate the rigid modes and strain modes all having a linear amplitude, we use the inner product in equation 31 and substitute $\mathbf{V}^{i}$ and $\mathbf{V}^{j}$ with $\mathbf{V}^{\mathrm{L}}$. As highlighted, modes of first order have either zero strain or constant strain. Consequently, we use the cross section displacement vectors $\mathbf{v}_{0 w}, \mathbf{v}_{0 \Omega}, \mathbf{v}_{1 w}$ and $\mathbf{v}_{1 \Omega}$ in the inner product (i.e. $k=l=0$ ). Hence, let us analyze this strain energy density of the four first order modes resulting in a $4 \times 4$ matrix $\mathbf{K}^{\mathrm{L}}$ as follows:

$$
\mathbf{K}^{\mathrm{L}}=\left\langle\left[\begin{array}{c}
\mathbf{V}_{0}^{\mathrm{L}} \\
\mathbf{V}_{1}^{\mathrm{L}}
\end{array}\right],\left[\begin{array}{c}
\mathbf{V}_{0}^{\mathrm{L}} \\
\mathbf{V}_{1}^{\mathrm{L}}
\end{array}\right]\right\rangle_{E}
$$

From the four dimensional subspace $\mathbf{K}^{\mathrm{L}}$ we can now extract eigenvalues and eigenvectors in order to identify and separate the rigid modes $\mathbf{V}^{\mathrm{L}_{R}}$ from the strain modes $\mathbf{V}^{\mathrm{L}_{E}}$. As a bonus the extensional and torsional modes are separated into individual principal eigenmodes as well. The rigid body motions have zero eigenvalues whereas extension and torsion modes have separate eigenvalues related to axial stiffness and torsional stiffness. The full polynomial vector space takes the following form:

$$
\mathbb{V}=\underbrace{\left[\begin{array}{cc|ccc}
\cdot & \cdot & \cdot & \cdot & \mathbf{V}_{3}^{\mathrm{S}} \\
\cdot & \cdot & \mathbf{V}_{2}^{\mathrm{B}} & \mathbf{V}_{2}^{\mathrm{S}} \\
\cdot & \mathbf{V}_{1}^{\mathrm{L}_{R}} & \mathbf{V}_{\text {Strain modes }}^{\mathbf{V}_{1}^{\mathrm{L}_{E}}} & \mathbf{V}_{1}^{\mathrm{B}} & \mathbf{V}_{1}^{\mathrm{S}} \\
\mathbf{V}_{0}^{\mathrm{C}} & \mathbf{V}_{0}^{\mathrm{L}_{R}} & \mathbf{V}_{0}^{\mathrm{L}_{E}} & \mathbf{V}_{0}^{\mathrm{B}} & \mathbf{V}_{0}^{\mathrm{S}}
\end{array}\right]}_{\text {Rigid modes }}
$$

b) Remove lower order strain modes from higher order strain modes

Since the higher order strain modes are contaminated by parts of lower order strain modes the next step is to remove this contamination. For this purpose, we will use a matrix projection procedure with an adequate inner product related to the energy. Since both the linear strain modes and the second order bending strain modes correspond to constant strain and constant strain energy density we will use the inner product defined in equation (31). Furthermore, we will use dots below a matrix symbol to indicate the updated matrix, however, in the following subsections with independent operations we will discard the dots of previous subsections. Thus, subtracting the linear strain modes from the bending modes can be done as shown here:

$$
\mathbf{V}^{\mathrm{B}}=\mathbf{V}^{\mathrm{B}}-\mathbf{V}^{\mathrm{L}_{E}} \mathcal{P}_{E}^{\mathrm{L}_{E} \mathrm{~B}}
$$

in which $\mathcal{P}_{E}^{\mathrm{L}_{E} \mathrm{~B}}$ is given by:

$$
\mathcal{P}_{E}^{\mathrm{L}_{E} \mathrm{~B}}=\left\langle\left[\begin{array}{c}
\mathbf{V}_{0}^{\mathrm{L}_{E}} \\
\mathbf{V}_{1}^{\mathrm{L}_{E}}
\end{array}\right],\left[\begin{array}{c}
\mathbf{V}_{0}^{\mathrm{L}_{E}} \\
\mathbf{V}_{1}^{\mathrm{L}_{E}}
\end{array}\right]\right\rangle_{E}^{-1}\left\langle\left[\begin{array}{c}
\mathbf{V}_{0}^{\mathrm{L}_{E}} \\
\mathbf{V}_{1}^{\mathrm{L}_{E}}
\end{array}\right],\left[\begin{array}{c}
\mathbf{V}_{0}^{\mathrm{B}} \\
\mathbf{V}_{1}^{\mathrm{B}}
\end{array}\right]\right\rangle_{E}
$$

The inner strain products in the angular brackets were defined in equation 31.

Similar, linear strain and second order bending are removed from the third order modes using:

$$
\mathbf{V}^{\mathrm{S}}=\mathbf{V}^{\mathrm{S}}-\left(\mathbf{V}^{\mathrm{L}_{E}} \mathcal{P}_{E}^{\mathrm{L}_{E} \mathrm{~S}}+\mathbf{V}^{\mathrm{B}} \mathcal{P}_{E}^{\mathrm{BS}}\right)
$$

where

$$
\mathcal{P}_{E}^{\mathrm{L}_{E} \mathrm{~S}}=\left\langle\left[\begin{array}{c}
\mathbf{V}_{0}^{\mathrm{L}_{E}} \\
\mathbf{V}_{1}^{\mathrm{L}_{E}}
\end{array}\right],\left[\begin{array}{c}
\mathbf{V}_{0}^{\mathrm{L}_{E}} \\
\mathbf{V}_{1}^{\mathrm{L}_{E}}
\end{array}\right]\right\rangle_{E}^{-1}\left\langle\left[\begin{array}{c}
\mathbf{V}_{0}^{\mathrm{L}_{E}} \\
\mathbf{V}_{1}^{\mathrm{L}_{E}}
\end{array}\right],\left[\begin{array}{c}
\mathbf{V}_{0}^{\mathrm{S}} \\
\mathbf{V}_{1}^{\mathrm{S}}
\end{array}\right]\right\rangle_{E}
$$

and

$$
\mathcal{P}_{E}^{\mathrm{BS}}=\left\langle\left[\begin{array}{c}
\mathbf{V}_{0}^{\mathrm{B}} \\
\mathbf{V}_{1}^{\mathrm{B}}
\end{array}\right],\left[\begin{array}{c}
\mathbf{V}_{0}^{\mathrm{B}} \\
\mathbf{V}_{1}^{\mathrm{B}}
\end{array}\right]\right\rangle_{E}^{-1}\left\langle\left[\begin{array}{c}
\mathbf{V}_{0}^{\mathrm{B}} \\
\mathbf{V}_{1}^{\mathrm{B}}
\end{array}\right],\left[\begin{array}{c}
\mathbf{V}_{0}^{\mathrm{S}} \\
\mathbf{V}_{1}^{\mathrm{S}}
\end{array}\right]\right\rangle_{E}
$$

Now we have made sure that the strain modes are pure, however, bending and shear modes are not in principal directions and the modes may also be contaminated by rigid modes.

\section{c) Find principal bending modes from the second order modes}

At the present stage we have sorted the modes into rigid modes and strain modes according to order. Pure extension and restrained torsion have been found. However, the bending modes and shear modes do not reflect bending about principal axes. Let us therefore extract a $2 \times 2$ stiffness matrix: $\mathbf{K}^{\mathrm{B}}$ from a bending subspace using the inner strain product in terms of equation (31) and the two second order modes in $\mathbf{V}^{\mathrm{B}}$, i.e.

$$
\mathbf{K}^{\mathrm{B}}=\left\langle\left[\begin{array}{c}
\mathbf{V}_{0}^{\mathrm{B}} \\
\mathbf{V}_{1}^{\mathrm{B}}
\end{array}\right],\left[\begin{array}{c}
\mathbf{V}_{0}^{\mathrm{B}} \\
\mathbf{V}_{1}^{\mathrm{B}}
\end{array}\right]\right\rangle_{E}
$$

From this two dimensional subspace, we now extract eigenvectors identifying the principal bending modes. Thereby we can find the bending modes related to the principal axes of classic theory. Eigenvalues are related to the bending stiffness's. 
Table 4: Pseudo mass matrices of cross section elements

$$
\begin{aligned}
& \mathbf{m}_{w w}^{e l}=\int_{0}^{b_{e l}}\left(t_{e l} \mathbf{N}_{n}^{\top} \mathbf{N}_{n}+t_{e l} \mathbf{N}_{s}^{\top} \mathbf{N}_{s}+\frac{1}{12} t_{e l}^{3} \mathbf{N}_{n, s}^{\top} \mathbf{N}_{n, s}\right) d s \\
& \mathbf{m}_{\Omega \Omega}^{e l}=\int_{0}^{b_{e l} l}\left(t_{e l} \mathbf{N}_{\Omega}^{\top} \mathbf{N}_{\Omega}+\frac{1}{12} t_{e l}^{3} \mathbf{N}_{\alpha}^{\top} \mathbf{N}_{\alpha}\right) d s
\end{aligned}
$$

\section{d) Find shear modes following the principal axis of bending}

The shear modes correspond to the third order modes, which have constant shear strain and linear varying bending shear. Thus, the bending terms are related to the quadratic energy terms $\left(z^{2}\right.$-terms). Let us therefore extract a $2 \times 2$ stiffness matrix: $\mathbf{K}^{\mathrm{S}}$ of the bending part of the third order (shear) subspace again using the inner strain product and the two third order modes as follows:

$$
\mathbf{K}^{\mathrm{S}}=\left\langle\left[\begin{array}{c}
\mathbf{V}_{1}^{\mathrm{S}} \\
\mathbf{V}_{2}^{\mathrm{S}}
\end{array}\right],\left[\begin{array}{c}
\mathbf{V}_{1}^{\mathrm{S}} \\
\mathbf{V}_{2}^{\mathrm{S}}
\end{array}\right]\right\rangle_{E}
$$

Notice that the inner product uses: $k=l=1$ in equation 31.

From the two dimensional stiffness subspace related to the linearly varying part of the shear modes we can extract eigenvectors that identify the principal bending modes and thus transform the third order shear modes in accordance with the principal directions of the bending modes of classic beam theory.

\section{e) Transform rigid modes according to principal directions}

The principal directions are now given by the translations of the quadratic variation of the bending modes $\mathbf{V}_{2}^{\mathrm{B}}$ or rather $\mathbf{V}_{2 w}^{\mathrm{B}}$, (since $\mathbf{V}_{2 \Omega}^{\mathrm{B}}$ can be disregarded due to its diminishing magnitude). Hence, through a matrix projection procedure, it is possible to rearrange the two linear rigid modes to follow the principal axes. As we are operating on a geometric basis, we formulate the projection through a geometric inner product anticipating that the displacements correspond to velocities of a pseudo inertial energy density with unit mass density being found as:

$$
\begin{aligned}
U_{I} & =\frac{1}{2} \int_{A} u_{n}^{i} u_{n}^{j}+u_{s}^{i} u_{s}^{j}+u_{z}^{i} u_{z}^{j} d A \\
& =\frac{1}{2}\left[\begin{array}{c}
\mathbf{v}_{w}^{i} \psi \\
\mathbf{v}_{\Omega}^{i} \eta
\end{array}\right]^{\top}\left[\begin{array}{cc}
\mathbf{M}_{w w} & \cdot \\
\cdot & \mathbf{M}_{\Omega \Omega}
\end{array}\right]\left[\begin{array}{c}
\mathbf{v}_{w}^{j} \psi \\
\mathbf{v}_{\Omega}^{j} \eta
\end{array}\right]
\end{aligned}
$$

where the pseudo mass matrices $\mathbf{M}_{w w}$ and $\mathbf{M}_{\Omega \Omega}$ are found by assembly of the cross section element mass matrices $\mathbf{m}_{w w}^{e l}$ and $\mathbf{m}_{\Omega \Omega}^{e l}$ given in table 4

A geometric inner product, "similar" to the one related to strains in equation (31), is formulated based on the inertial energy $U_{I}$ substituting the displacement modes from equation (29). Then, a geometric inner product is formulated as:

$$
\left\langle\mathbf{V}_{k}^{i}, \mathbf{V}_{l}^{j}\right\rangle_{I}=\left[\begin{array}{c}
\mathbf{v}_{k w}^{i} \\
\mathbf{v}_{k \Omega}^{i}
\end{array}\right]^{\top}\left[\begin{array}{cc}
\mathbf{M}_{w w} & \cdot \\
\cdot & \mathbf{M}_{\Omega \Omega}
\end{array}\right]\left[\begin{array}{c}
\mathbf{v}_{l w}^{j} \\
\mathbf{v}_{l \Omega}^{j}
\end{array}\right]
$$

where $i$ and $j$ indicates a polynomial family and $k$ and $l$ represents a coefficient number.

Using principles from matrix projection we transform the linear modes using a subspace spanned by the second order parts of the bending modes. The linear modes following principal directions are then found through:

$$
\mathbf{V}^{\mathrm{L}_{R}}=\mathbf{V}^{\mathrm{L}_{R}} \mathcal{P}_{I}^{\mathrm{L}_{R} \mathrm{~B}}
$$

where

$$
\mathcal{P}_{I}^{\mathrm{L}_{R} \mathrm{~B}}=\left\langle\mathbf{V}_{1}^{\mathrm{L}_{R}}, \mathbf{V}_{1}^{\mathrm{L}_{R}}\right\rangle_{I}^{-1}\left\langle\mathbf{V}_{1}^{\mathrm{L}_{R}}, \mathbf{V}_{2}^{\mathrm{B}}\right\rangle_{I}
$$

In a similar manner, it is possible to transform the rigid constant modes into principal directions. As a matter of fact, the four directions are already represented in the solution space being the first order part of the linear mode family. Thus, through a matrix projection the constant modes are projected onto the directions given in $\mathbf{V}_{1}^{\mathrm{L}}$. So,

$$
\mathbf{V}^{\mathrm{C}}=\mathbf{V}^{\mathrm{C}} \mathcal{P}_{I}^{\mathrm{CL}}
$$

where

$$
\mathcal{P}_{I}^{\mathrm{CL}}=\left\langle\mathbf{V}_{0}^{\mathrm{C}}, \mathbf{V}_{0}^{\mathrm{C}}\right\rangle_{I}^{-1}\left\langle\mathbf{V}_{0}^{\mathrm{C}}, \mathbf{V}_{1}^{\mathrm{L}}\right\rangle_{I}
$$

With the above transformation the rigid modes are now directed according to the principal directions.

\section{f) Remove lower order rigid modes from higher order modes}

The last modification step missing is to make sure that no higher order modes contain any constant or linear rigid motions.

While the rigid body modes have zero strains we cannot use the procedure from step $b$ ) and the strain energy density. As a consequence, we need to introduce a separate inner product to remove the contamination in the higher order modes. Hence we use the geometric inner product from equation 42 .

First, let us clean the linear rigid modes, i.e. let us remove the contamination with constant rigid modes from the linear rigid modes by:

$$
\mathbf{V}^{\mathrm{L}_{R}}=\mathbf{V}^{\mathrm{L}_{R}}-\mathbf{V}^{\mathrm{C}} \mathcal{P}_{I}^{\mathrm{CL}_{R}}
$$

with

$$
\mathcal{P}_{I}^{\mathrm{CL}}=\left\langle\mathbf{V}_{0}^{\mathrm{C}}, \mathbf{V}_{0}^{\mathrm{C}}\right\rangle_{I}^{-1}\left\langle\mathbf{V}_{0}^{\mathrm{C}}, \mathbf{V}_{0}^{\mathrm{L}_{R}}\right\rangle_{I}
$$

Secondly, let us clean the linear strain modes, i.e. let us remove the constant rigid modes from the linear strain modes:

$$
\mathbf{V}^{\mathrm{L}_{E}}=\mathbf{V}^{\mathrm{L}_{E}}-\mathbf{V}^{\mathrm{C}} \mathcal{P}_{I}^{\mathrm{CL}_{E}}
$$

with

$$
\mathcal{P}_{I}^{\mathrm{CL}_{E}}=\left\langle\mathbf{V}_{0}^{\mathrm{C}}, \mathbf{V}_{0}^{\mathrm{C}}\right\rangle_{I}^{-1}\left\langle\mathbf{V}_{0}^{\mathrm{C}}, \mathbf{V}_{0}^{\mathrm{L}_{E}}\right\rangle_{I}
$$

Then, we clean the second order modes:

$$
\mathbf{V}^{\mathrm{B}}=\mathbf{V}^{\mathrm{B}}-\left(\mathbf{V}^{\mathrm{C}} \mathcal{P}_{I}^{\mathrm{CB}}+\mathbf{V}^{\mathrm{L}_{R}} \mathcal{P}_{I}^{\mathrm{L}_{R} \mathrm{~B}}\right)
$$


with

$$
\mathcal{P}_{I}^{\mathrm{CB}}=\left\langle\mathbf{V}_{0}^{\mathrm{C}}, \mathbf{V}_{0}^{\mathrm{C}}\right\rangle_{I}^{-1}\left\langle\mathbf{V}_{0}^{\mathrm{C}}, \mathbf{V}_{0}^{\mathrm{B}}\right\rangle_{I}
$$

and

$$
\mathcal{P}_{I}^{\mathrm{L}_{R} \mathrm{~B}}=\left\langle\mathbf{V}_{1}^{\mathrm{L}_{R}}, \mathbf{V}_{1}^{\mathrm{L}_{R}}\right\rangle_{I}^{-1}\left\langle\mathbf{V}_{1}^{\mathrm{L}_{R}}, \mathbf{V}_{1}^{\mathrm{B}}\right\rangle_{I}
$$

Finally, we clean the third order modes through:

$$
\mathbf{V}^{\mathrm{S}}=\mathbf{!}^{\mathrm{S}}-\left(\mathbf{V}^{\mathrm{C}} \mathcal{P}_{I}^{\mathrm{CS}}+\mathbf{!}^{\mathrm{L}_{R}} \mathcal{P}_{I}^{\mathrm{L}_{R} \mathrm{~S}}\right)
$$

with

$$
\mathcal{P}_{I}^{\mathrm{CS}}=\left\langle\mathbf{V}_{0}^{\mathrm{C}}, \mathbf{V}_{0}^{\mathrm{C}}\right\rangle_{I}^{-1}\left\langle\mathbf{V}_{0}^{\mathrm{C}}, \mathbf{V}_{0}^{\mathrm{S}}\right\rangle_{I}
$$

and

$$
\mathcal{P}_{I}^{\mathrm{L}_{R} \mathrm{~S}}=\left\langle\mathbf{V}_{1}^{\mathrm{L}_{R}}, \mathbf{V}_{1}^{\mathrm{L}_{R}}\right\rangle_{I}^{-1}\left\langle\mathbf{V}_{1}^{\mathrm{L}_{R}}, \mathbf{Y}_{1}^{\mathrm{S}}\right\rangle_{I}
$$

\section{Displacement formulation of polynomial modes}

Having completed the steps $a$ ) through $f$ ) we can assemble the fundamental polynomial solutions into a practical displacement formulation. For us a practical displacement formulation allows the use of the Hadarmard product in which the amplitude functions are in the form of a diagonal matrix. Let us therefore introduce a polynomial displacement vector $\mathbf{V}_{p}$ consisting of the four block matrices of $\mathbb{V}$ in a row of blocks:

$$
\mathbf{V}_{p}=\left[\begin{array}{llll}
\mathbf{V}_{3} & \mathbf{V}_{2} & \mathbf{V}_{1} & \mathbf{V}_{0}
\end{array}\right]
$$

Furthermore, let us introduce $\mathbf{I}_{p}$ as a $n_{z} \times n_{z}=12 \times 12$ diagonal unit matrix. This allows us to write the displacement formulation of the fundamental polynomial modes as follows:

$$
\begin{aligned}
\mathbf{u}_{p} & =\left[\begin{array}{c}
\mathbf{V}_{3} \\
\mathbf{V}_{2} \\
\mathbf{V}_{1} \\
\mathbf{V}_{0}
\end{array}\right]^{\top}\left[\begin{array}{cccc}
\frac{1}{6} z^{3} \mathbf{I}_{p} & \cdot & \cdot & \cdot \\
\cdot & \frac{1}{2} z^{2} \mathbf{I}_{p} & \cdot & \cdot \\
\cdot & \cdot & z \mathbf{I}_{p} & \cdot \\
\cdot & \cdot & \cdot & \mathbf{I}_{p}
\end{array}\right]\left[\begin{array}{c}
\mathbf{I}_{p} \\
\mathbf{I}_{p} \\
\mathbf{I}_{p} \\
\mathbf{I}_{p}
\end{array}\right] \mathbf{c}_{p} \\
& =\mathbf{V}_{p} \mathbf{\Psi}_{p} \mathbf{T}_{p} \mathbf{c}_{p}
\end{aligned}
$$

where we have taken the liberty to introduce the diagonal matrix $\boldsymbol{\Psi}_{p}$ that contains all polynomial amplitude functions and a polynomial transformation matrix $\mathbf{T}_{p}$. The column vector $\mathbf{c}_{p}$ holds the $n_{z}=12$ constants belonging to each fundamental displacement mode. These constants are the constants belonging to each of the (mode) solutions to the differential equations and are to be determined by the use of boundary conditions.

\subsection{Full displacement formulation}

Finally, the full displacement solution encompassing both exponential and fundamental polynomial modes can be found. The exponential modes found in equation (19) may also be written as:

$$
\mathbf{u}_{e}=\mathbf{V}_{e} \Psi_{e} \mathbf{I}_{e} \mathbf{c}_{e}
$$

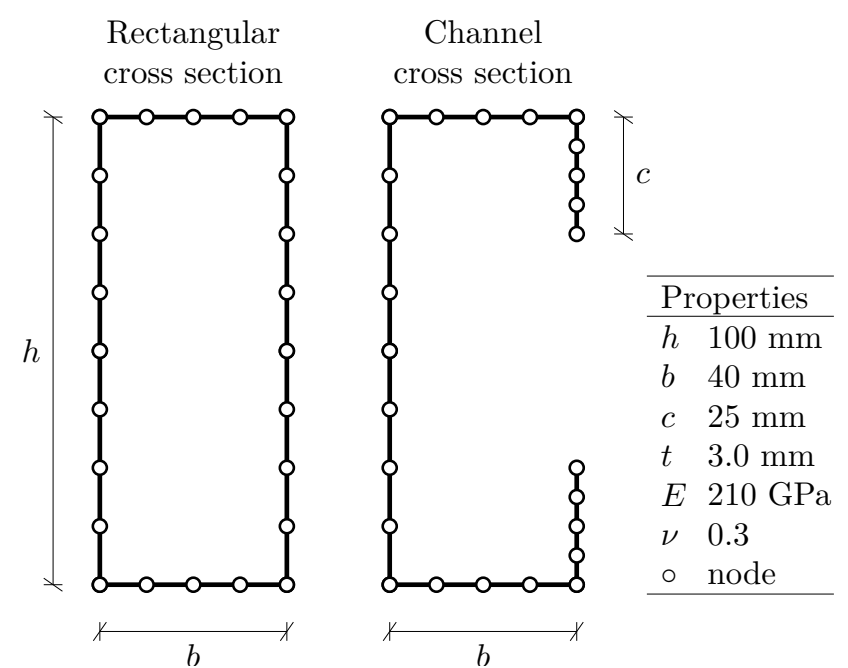

Figure 5: Geometrical and material properties of the rectangular cross section and the lipped channel cross section.

where $\mathbf{I}_{e}$ is just a "dummy" unit matrix of size $n_{e} \times n_{e}$ that allows us to write the full displacement solution as:

$$
\begin{aligned}
\mathbf{u} & =\mathbf{u}_{p}+\mathbf{u}_{e}=\mathbf{V} \boldsymbol{\Psi} \mathbf{T}_{c} \mathbf{c} \\
& =\left[\begin{array}{ll}
\mathbf{V}_{p} & \mathbf{V}_{e}
\end{array}\right]\left[\begin{array}{cc}
\boldsymbol{\Psi}_{p} & \cdot \\
\cdot & \boldsymbol{\Psi}_{e}
\end{array}\right]\left[\begin{array}{cc}
\mathbf{T}_{p} & \cdot \\
\cdot & \mathbf{I}_{e}
\end{array}\right]\left[\begin{array}{l}
\mathbf{c}_{p} \\
\mathbf{c}_{e}
\end{array}\right]
\end{aligned}
$$

In which we have introduced $\mathbf{V}$ as the full mode matrix, $\boldsymbol{\Psi}$ as the full diagonal amplitude matrix and $\mathbf{T}_{c}$ as a constant transformation matrix containing $\mathbf{T}_{p}$ and $\mathbf{I}_{e}$. This concludes the cross section mode determination procedure. Note that since each fundamental mode is a combination of four cross section displacement fields $\mathbf{v}_{1}^{i}$ to $\mathbf{v}_{4}^{i}$ the number of columns in $\mathbf{V}$ is: $4 n_{z}+\left(2 n-n_{z}\right)=4 n_{z}+n_{e}$, where $n$ is the number of degrees of freedom in the cross section, $n_{z}$ is the number of fundamental modes, and $n_{e}$ the number of exponential modes. However, the number of constants c is $2 n=n_{z}+n_{e}$.

\section{Results of the mode determination procedure}

Using the approach just presented within this paper, it is possible to identify all eigenmodes of a thin-walled beam with an arbitrary shaped cross section, based on simple elastic constitutive relations and an appropriate discritization of the cross section. In the following, results from the mode determination procedure will be presented for a rectangular cross section and a channel cross section. The cross section discretization and geometrical as well as material properties are shown in figure 5 for both cross sections. In the rectangular cross section analysed, there are 24 nodes with six degrees of freedom each giving a total of $2 \cdot 144$ modes of which 12 are the fundamental modes. In the channel cross section analysed, there are 25 nodes with six degrees of freedom resulting in a total of $2 \cdot 150$ deformation modes. 
Table 5: First twenty-two non-null eigenvalues, i.e. $\lambda \neq 0$

\begin{tabular}{l|rr}
\hline & Rectangular cross section & Channel cross section \\
\hline 13 & $0.0065+0.0060 i$ & 0.0011 \\
14 & $0.0065-0.0060 i$ & -0.0011 \\
15 & $-0.0065+0.0060 i$ & $0.0058+0.0046 i$ \\
16 & $-0.0065-0.0060 i$ & $0.0058-0.0046 i$ \\
17 & $0.0363+0.0144 i$ & $-0.0058+0.0046 i$ \\
18 & $0.0363-0.0144 i$ & $-0.0058-0.0046 i$ \\
19 & $-0.0363+0.0144 i$ & $0.0079+0.0067 i$ \\
20 & $-0.0363-0.0144 i$ & $-0.0079+0.0067 i$ \\
21 & $0.0366+0.0136 i$ & $-0.0079-0.0067 i$ \\
22 & $0.0366-0.0136 i$ & 0.0373 \\
23 & $-0.0366+0.0136 i$ & -0.0373 \\
24 & $-0.0366-0.0136 i$ & -0.0388 \\
25 & $0.0374+0.0186 i$ & $0.0374+0.0170 i$ \\
26 & $0.0374-0.0186 i$ & $0.0374+0.0170 i$ \\
27 & $-0.0374+0.0186 i$ & $-0.0374+0.0170 i$ \\
28 & $-0.0374-0.0186 i$ & $-0.0374-0.0170 i$ \\
29 & $0.0401+0.0193 i$ & $0.0394+0.0164 i$ \\
30 & $0.0401-0.0193 i$ & $0.0394+0.0164 i$ \\
31 & $-0.0401+0.0193 i$ & $0.0394+0.0164 i$ \\
32 & $-0.0401-0.0193 i$ & $0.0394+0.0164 i$ \\
33 & 0.0616 & \\
34 & -0.0616 &
\end{tabular}

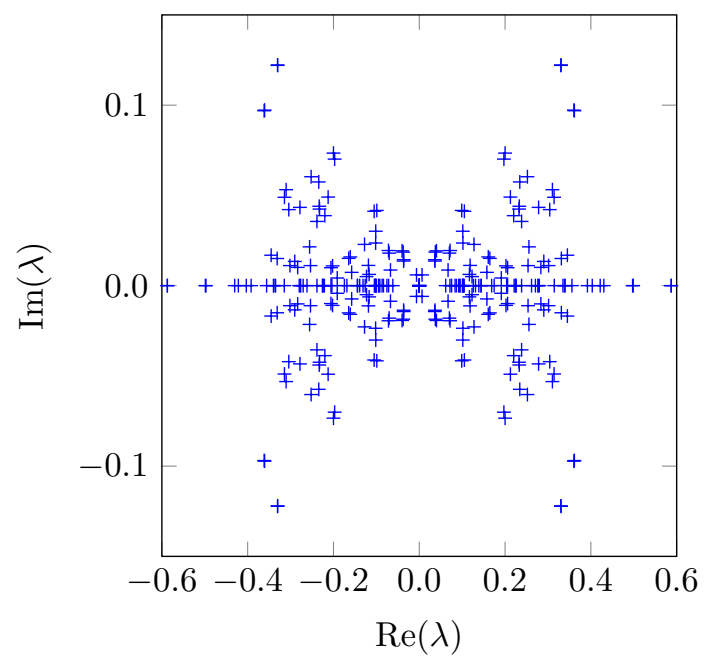

Figure 6: Plot of eigenvalues for the rectangular cross section

\subsection{Exponential modes}

To find the exponential modes we solve the quadratic eigenvalue problem in equation (18) using the state vector approach. This results in a pool of eigenvalues of which twelve are null, some are real pairs and others are complex quadruples. The first twenty-two non-null eigenvalues are listed in table 5 for both cross sections. These eigenvalues may be presented in the complex plane as shown in figure 6 for the rectangular cross section and in figure 7 for the channel cross section. From these figures, it is seen how the pairs of real eigenvalues $\{\lambda,-\lambda\}$ lay on the horizontal axis with $\operatorname{Im}(\lambda)=0$, whereas the complex quadruple eigenvalues, i.e. pairs of complex conjugated eigenvalues, $\{\lambda, \bar{\lambda},-\lambda,-\bar{\lambda}\}$, are those points which are distributed double-symmetrically in the complex plane. The eigenvalues can be seen as an inverse length scale related to the attenuation for the real part and to the period of the harmonic variations for the imaginary part. The first three sets of related amplitude functions are shown in figure 8 for the rectangular cross section and figure 9 for the channel cross section. Each row in these figures corresponds to either a couple or quadruple set of eigenvalues in which we use: $f(z)=e^{\lambda z}$ for eigenvalues with a negative real part and: $f(z)=e^{\lambda(z-\ell)}$ for eigenvalues with a positive real part, where $\ell$ is the beam length. These functions are also used in the amplitude matrix to limit the numerical magnitude of the exponential function. It can be seen how the imaginary parts yield positive or negative oscillation along the beam axis.

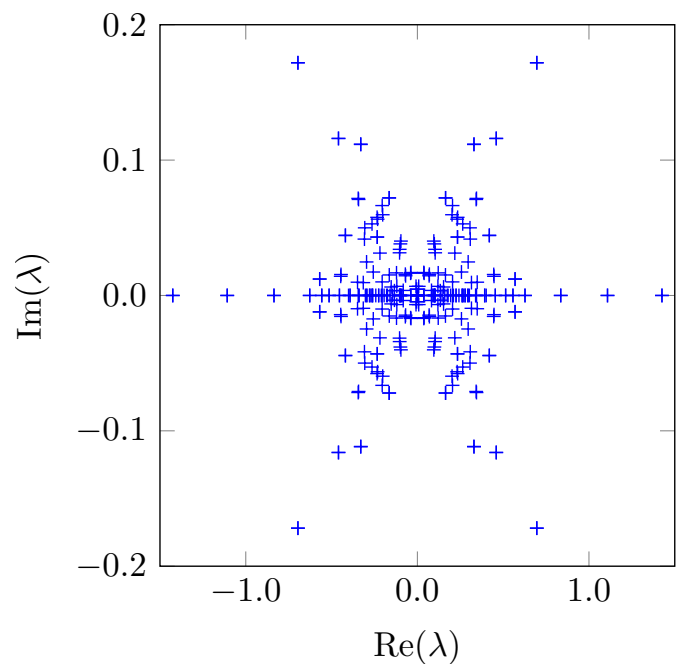

Figure 7: Plot of eigenvalues for the channel cross section 

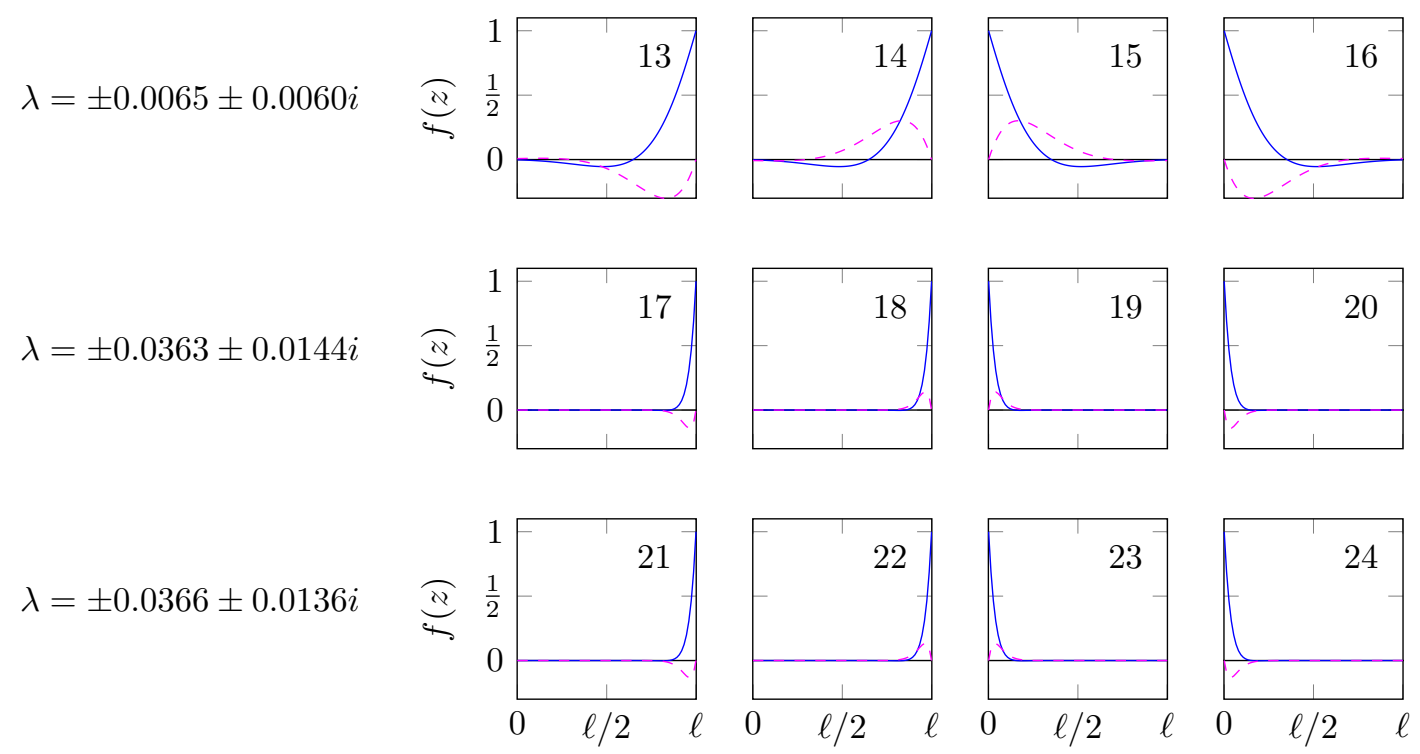

Real part

- - - - Imaginary part

$$
f(z)=e^{\lambda(z-\ell)}
$$

$f(z)=e^{\lambda z}$

Figure 8: Amplitude functions for rectangular cross section eigenmodes (with a beam length of $\ell=750 \mathrm{~mm}$ )

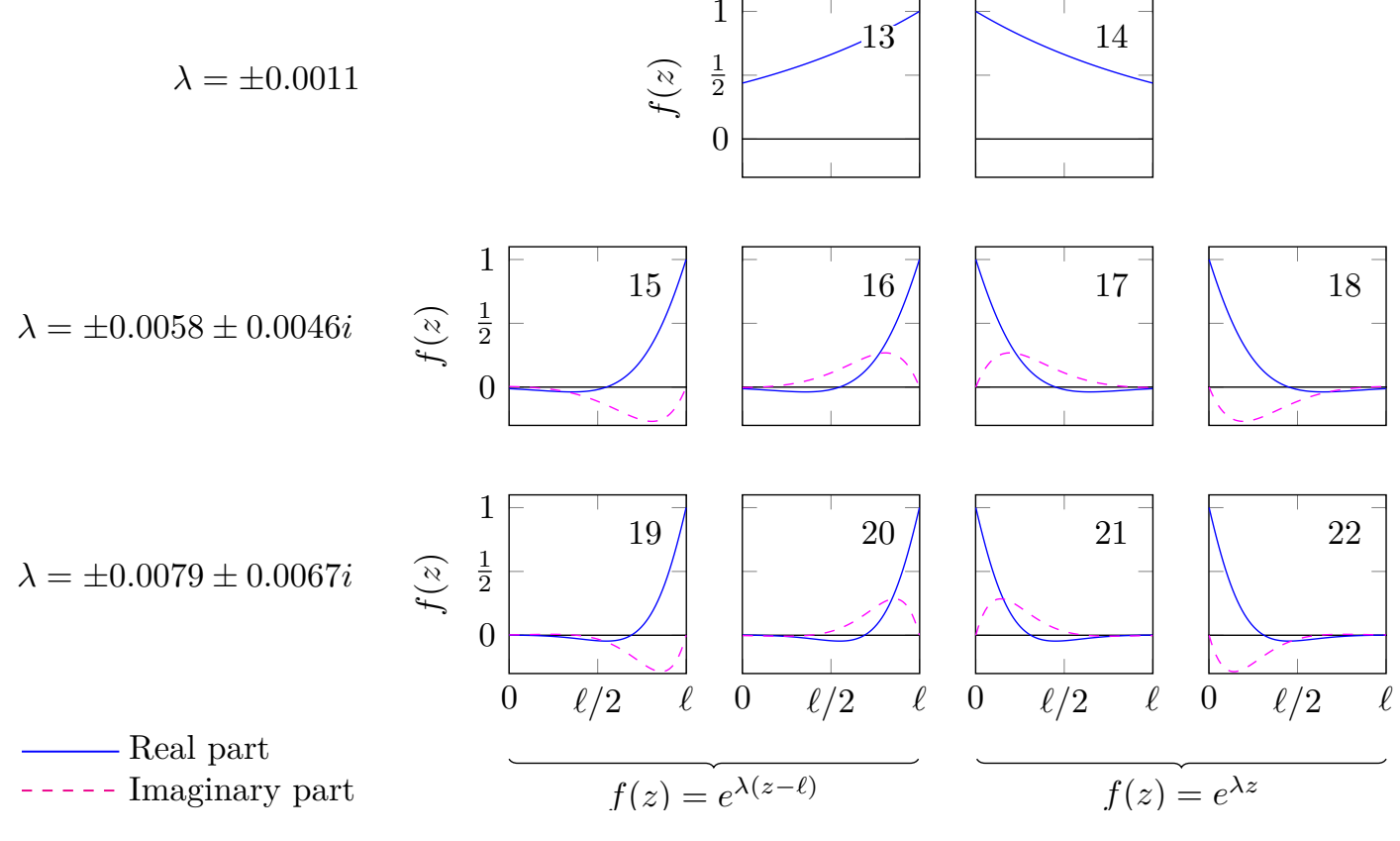

Figure 9: Amplitude functions for channel cross section eigenmodes (with a beam length of $\ell=750 \mathrm{~mm}$ ) 
Figures 10 and 11 illustrates the first 22 exponential displacement modes of the two cross sections. The displacement modes are separated into transverse displacements and warping displacements and the modes are ordered according to their attenuation length in an increasing order. As mentioned the modes are normalized in such a way that the real, largest displacement in either the $X$ or $Y$-direction is set to unity for the transverse displacements. To be able to see the imaginary part of a mode it is scaled by a factor "scale" given below the illustration. The magnitude of the warping part of a mode depends on the normalization of the transverse displacement. We therefore need to scale the warping modes by the ratio between maximum transverse displacement and maximum warping displacement to be able to plot them corresponding to a unit out of plane deformation. Hence, the "ratio" is given in the figure below the real part of the warping mode. We also need to separately "scale" the imaginary parts of the warping displacements - also given as a "scale".

For the channel cross section it is worth noting that the first mode with the longest attenuation length is the classic exponential part of restrained torsion, i.e. with rotation and warping of the cross section (giving normal stresses). Furthermore, it is notable that the rectangular cross section does not have this kind of mode and therefore restrained torsion of the rectangular cross section will involve distortion of the cross section as well.

The quadruple complex exponential modes can be transformed into four coupled real modes with a procedure presented by Jönsson and Andreassen [14]. To illustrate this two of these four modes with attenuation towards the other end are shown in figures $12 \mathrm{a}$ and $12 \mathrm{~b}$ for the rectangular cross section mode 13 through 14 and in figures $12 \mathrm{c}$ and $12 \mathrm{~d}$ for the channel cross section modes 19 through 20 . In both cases a beam length of $\ell=750 \mathrm{~mm}$ has been used for the illustration.

It can be seen that the eigenvalues that come in pairs are associated displacement modes, which have one cross section displacement field which attenuates in one or the other axial direction. Furthermore, the quadruple eigenvalues correspond to complex displacement modes which consist of one real cross section displacement field plus or minus one imaginary cross section displacement field, which may be combined corresponding to two different types of attenuations, one in each direction. Thus, the modes of the quadruple complex eigenvalues correspond to two cross section deformation fields, which are combined with the amplitude functions into four different spatial beam displacement modes.

\subsection{Fundamental modes}

The fundamental modes are found as the null space of $\mathbb{K}$ being the set of equilibrium equations to be fulfilled by the polynomial displacement modes up to third order. In the null space we then find modes of different polynomial order using SVD on the block related to a given order. Then we follow the procedure $a$ ) through $f$ ) to separate rigid body modes and strain modes and to identify the different fundamental modes illustrated in figure 4. The figures 13 and 14 illustrates these modes by only showing the relevant, either transverse displacement field or the warping displacement field depending on which is largest. In our case one of these always diminishes by a factor of about $10^{-3}$ or less. Furthermore we do not show displacements which are numerically so small that they are irrelevant, i.e. in our case a factor of $10^{-12}$ lower than the relevant part of the mode (dependent on machine precision). All displacement plots have been scaled to a maximum unit displacement. Therefore, for example in the illustration of a pure bending mode 9 in figure 13 or 14 the magnitude of the constant transverse displacements $\mathbf{v}_{0}^{9}$ related to the Poisson effect is in reality much smaller than shown compared to the quadratic transverse displacements $\mathbf{v}_{2}^{9}$.

It is very interesting, from an engineering point of view, to take a closer look at these cross section displacement fields. It can be seen that there are twelve fundamental modes combining twelve independent cross section displacement fields. The independent cross section displacement fields are identified in the bottom line of the figures corresponding to $\mathbf{V}_{0}$. Thus, with the theory of this paper the fundamental polynomial displacements of a thinwalled beam is described by twelve cross section displacement fields and not the conventional six or seven cross section displacement fields of three dimensional beam theory or three dimensional Vlasov beam theory, respectively. The six cross section displacement fields of classic beam theory are the 3 warping modes i.e. axial extension + two flexural and 3 transverse modes corresponding to pure axial extension and two flexural

\section{Conclusion}

The theory behind the novel prismatic thin-walled beam model including deformable cross sections, shear deformations and the Poisson effect has been introduced. Furthermore, a new mode determination procedure for determination of both exponential distortional displacement modes and the fundamental beam displacement modes has been presented and the modes have been illustrated in the results section. The new developments are especially related to the procedure used in determining and separating the fundamental modes mathematically and numerically. The novelty of the procedure lies in the use of both strain energy and the geometric based products relating to the order of the polynomial terms within each of the fundamental modes. Furthermore, the exponential modes presented do not decouple the original coupled quadratic differential equations, but they represent the full solution space, and decouple a related set of first order differential equations, as in Jönsson and Andreassen [14. However, the shear and Poisson effects are now included in all modes of the present formulation and the fundamental modes are not simple solutions based on additional constraint assumptions. 


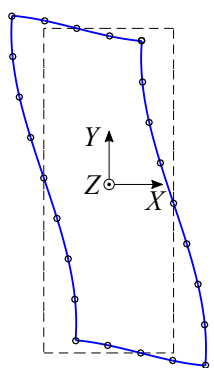

$\operatorname{Re}(13-16)$

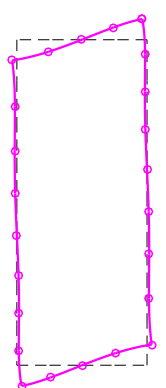

$\operatorname{Im}(13-16)$

scale: 31.4
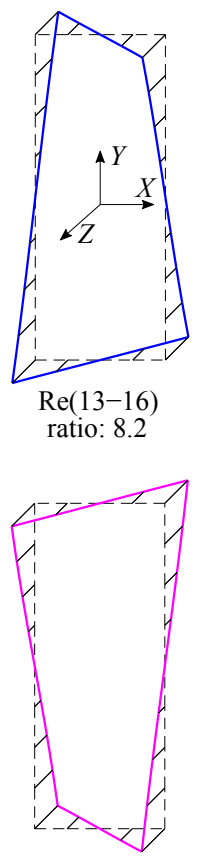

$\operatorname{Im}(13-16)$

scale: 0.99
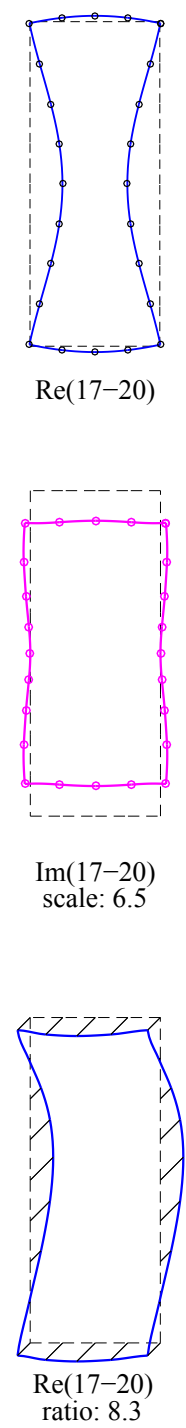

$\operatorname{Re}(17-20)$

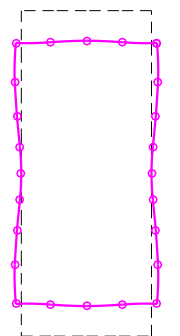

$\operatorname{Im}(17-20)$

scale: 6.5

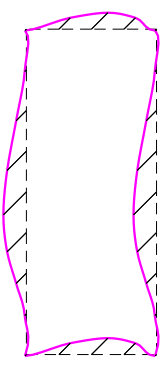

$\operatorname{Im}(17-20)$

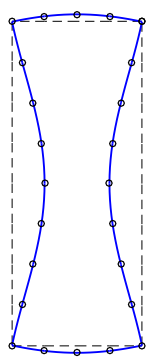

$\operatorname{Re}(21-24)$

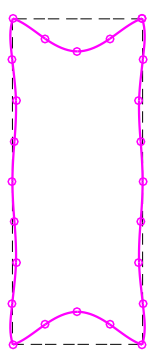

$\operatorname{Im}(21-24)$

(a) Transverse displacements
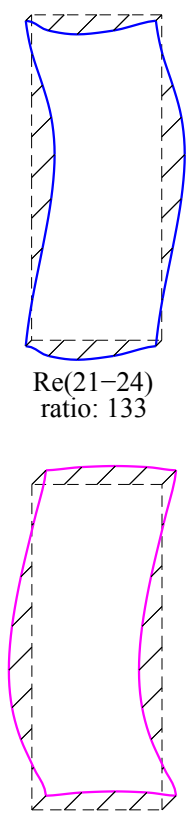

$\operatorname{Im}(21-24)$

scale: 1.0

$\operatorname{Re}(25-28)$

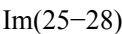

scale: 27.4

scale: 0.59
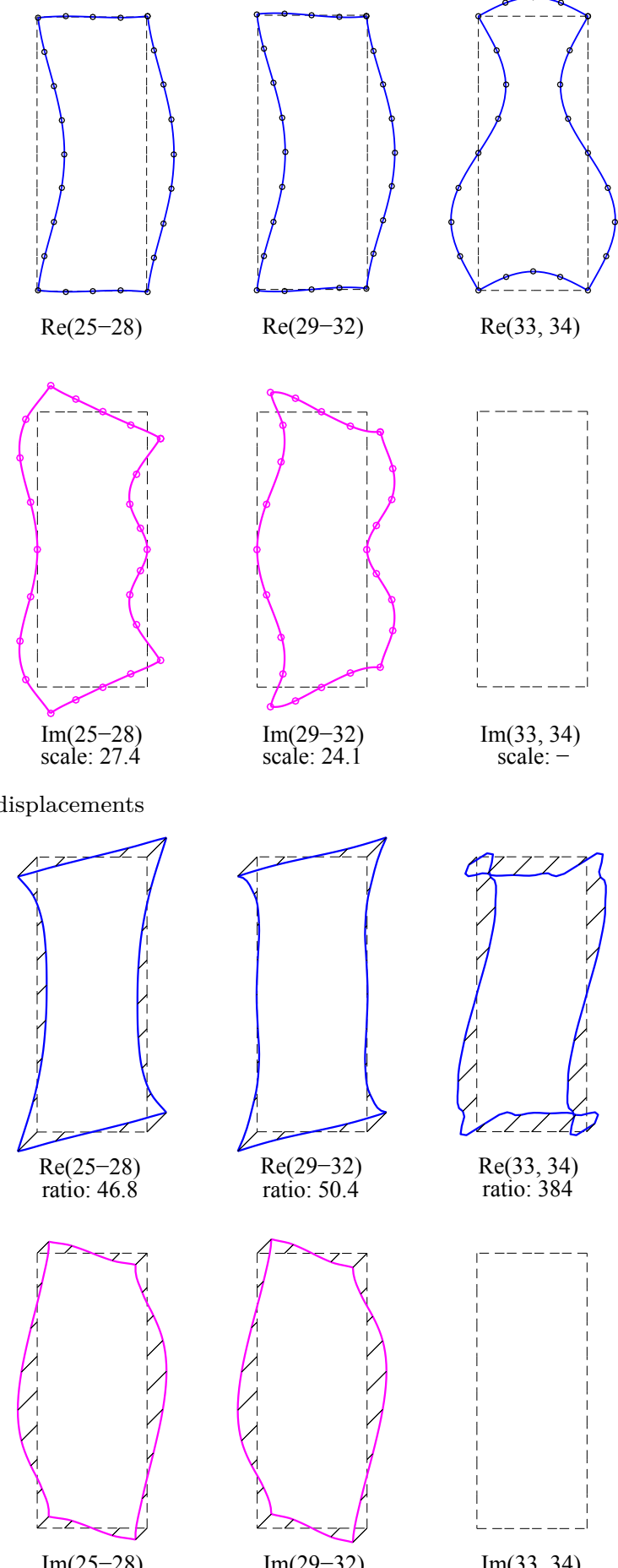

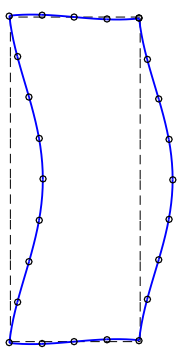

$\operatorname{Re}(29-32)$

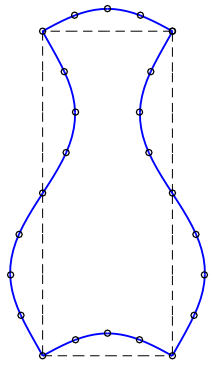

$\operatorname{Re}(33,34)$

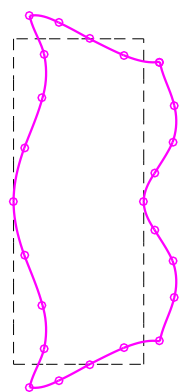

$\operatorname{Im}(29-32)$

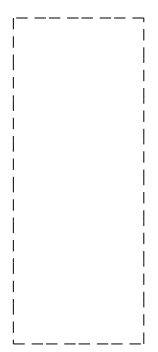

$\operatorname{Im}(33,34)$
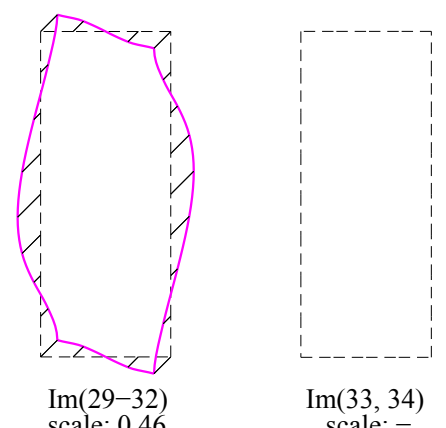

(b) Warping displacements

Figure 10: Exponential modes - Rectangular cross section

All the classic beam deformation modes have been found by the procedure and it is interesting and worth noting that restrained torsion is represented among the exponential solutions as a pure twist of open cross sections (such as the channel cross section) with the same warping function shape as the fundamental solution of unrestrained torsion.
Specially noteworthy is it that for closed cross sections, this is not the case.

It is also noteworthy, by observation of figures 13 and 14. that it seems that the twelve fundamental solutions being combinations of one to four different cross section displacement fields are simple combinations of twelve cross 


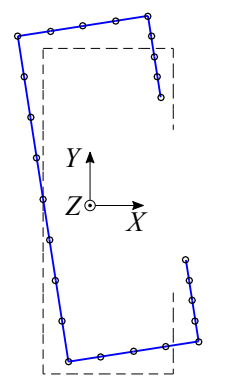

$\operatorname{Re}(13,14)$

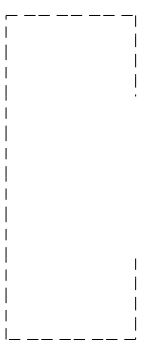

$\operatorname{Im}(13,14)$ scale: -

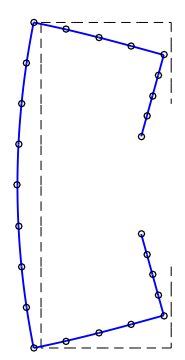

$\operatorname{Re}(15-18)$

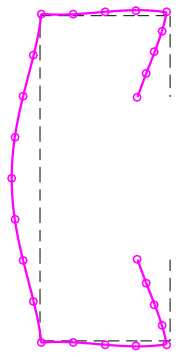

$\operatorname{Im}(15-18)$ scale: 60.2

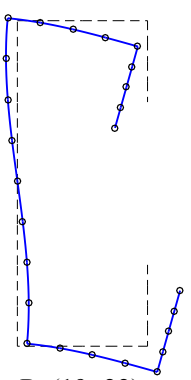

$\operatorname{Re}(19-22)$

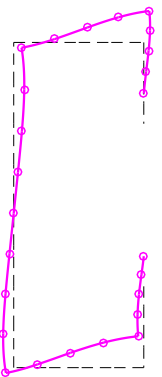

Im(19-22)

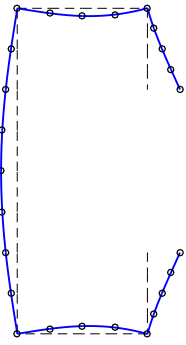

$\operatorname{Re}(23,24)$

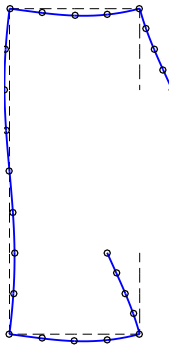

$\operatorname{Re}(25,26)$

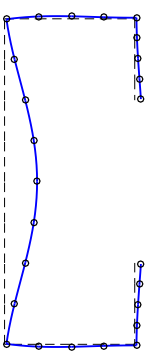

$\operatorname{Re}(27-30)$

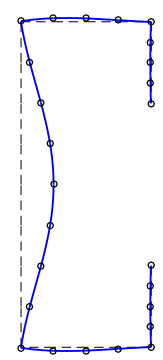

$\operatorname{Re}(31-34)$
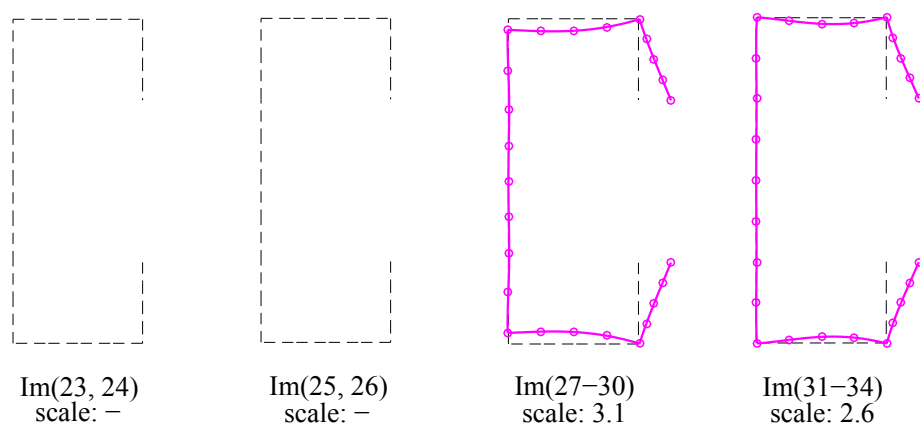

$\operatorname{Im}(27-30)$ scale: 3.1

$\operatorname{Im}(31-34)$

$\begin{array}{cc}\operatorname{Im}(23,24) & \operatorname{Im}(25,26) \\ \text { scale: }- & \text { scale: }-\end{array}$

(a) Transverse displacements

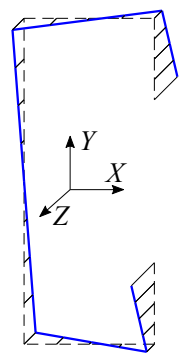

$\operatorname{Re}(13,14)$

ratio: 24.3

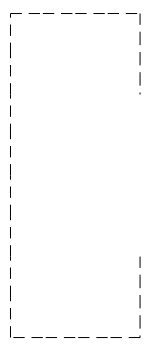

$\operatorname{Im}(13,14)$

scale: -

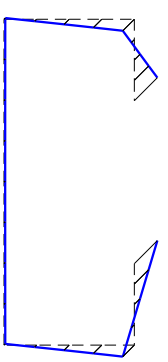

$\operatorname{Re}(15-18)$

ratio: 10.4

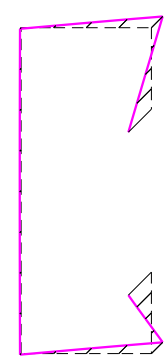

$\operatorname{Im}(15-18)$

scale: 1.2
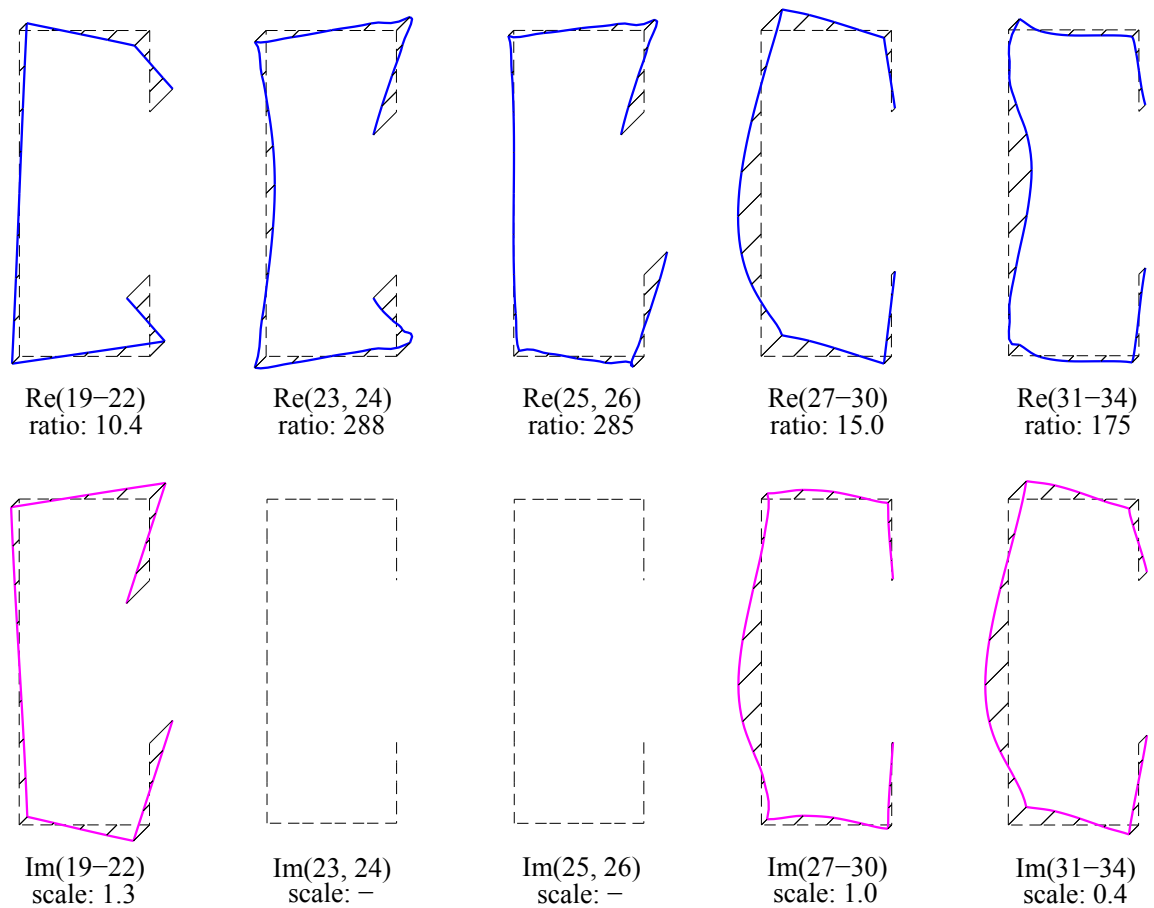

(b) Warping displacements

Figure 11: Exponential modes - Channel cross section

section displacement fields.

It is interesting that the defined internal strain energy products also can be used to find the principal direction of bending thereby giving a direct link to classic beam theory and perhaps also to the cross section properties. This link and the relation of the boundary conditions to the classic section forces of beam theory is the focus of the continued research. With the determination of all the displacement modes it is now possible to continue the research and formulate an advanced beam element based on exact axial 


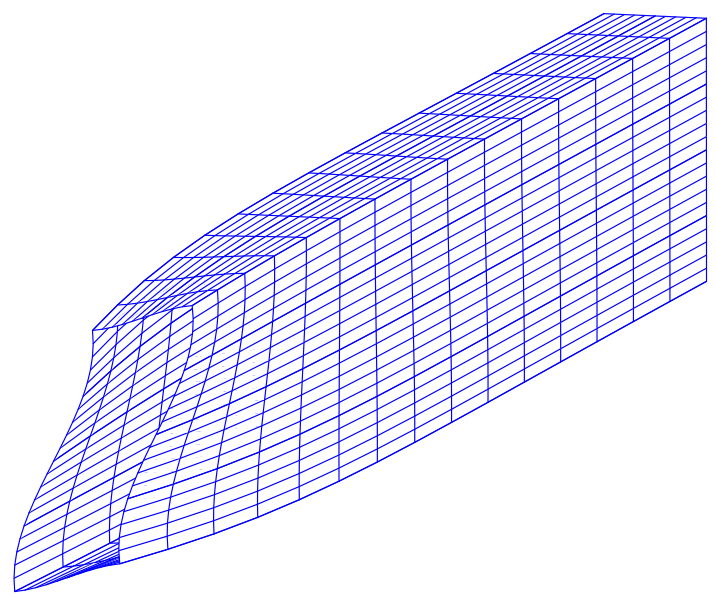

(a) The first real combination of mode 13 and 14 with respect to the rectangular cross section

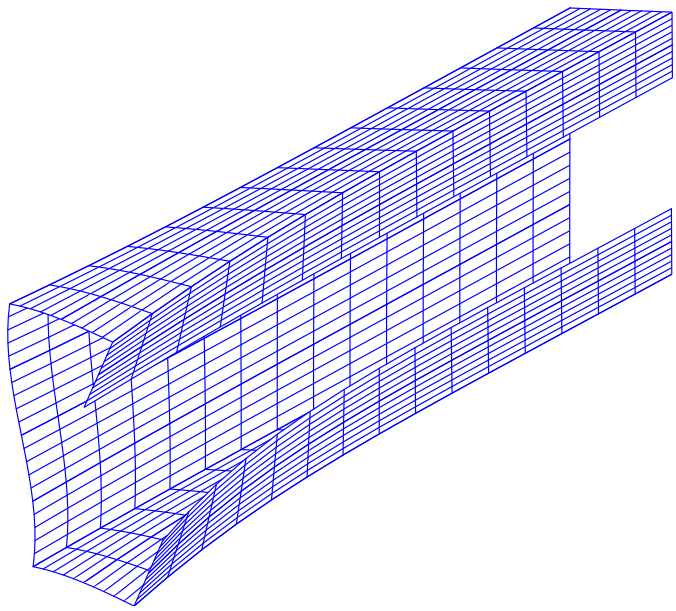

(c) The first real combination of mode 19 and 20 regarding the channel section

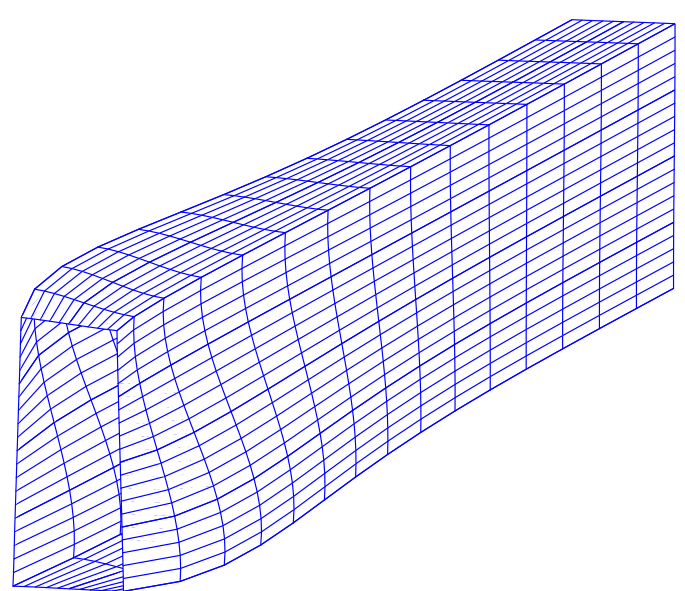

(b) The second real combination of mode 13 and 14 with respect to the rectangular cross section

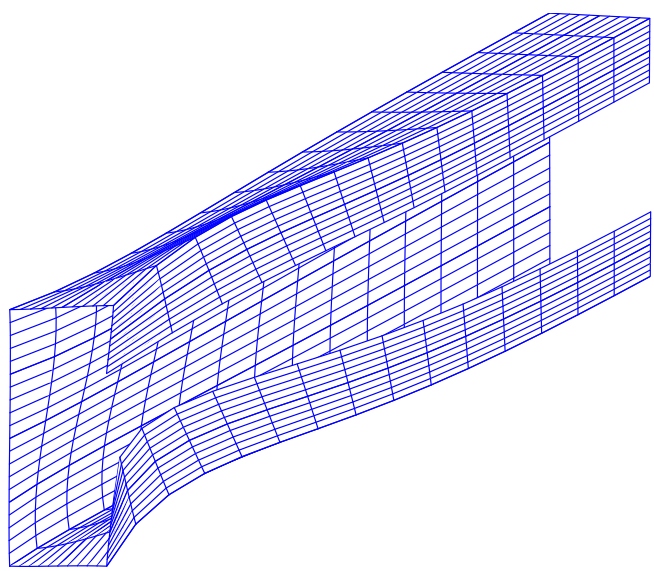

(d) The second real combination of mode 19 and 20 regarding the channel section

Figure 12: Quadruple complex distortional eigenmodes illustrated as real coupled modes

shape functions and the possibility of just choosing a reduced number of displacement modes.

\section{Acknowledgement}

The work presented in this paper has been supported financially by the Danish engineering consultancy NIRAS $\mathrm{A} / \mathrm{S}$ and the Innovation Fund Denmark by grant: 518900005B.

\section{References}

[1] V. Z. Vlasov, Thin-Walled Elastic Beams, 2nd Edition, Jerusalem, 1961, israel Program for scientific translations.

[2] R. Schardt, Eine erweiterung der technishen biegelehre für die berechnung biegestreifer prismatischer falfwerke, Der Stahlbau 35 (1966) 161-171.

[3] R. Schardt, Verallgemeinerte Technische Biegetheorie - Band 1 , Lineare Theorie, 2nd Edition, Metrum-Verlag,Darmstadt, 1989.

[4] J. M. Davies, P. Leach, First-order generalised beam theory, Journal of Constructional Steel Research 31 (1994) 187-220. doi : 10.1016/0143-974X(94)90010-8
[5] J. M. Davies, P. Leach, D. Heinz, Second-order generalised beam theory, Journal of Constructional Steel Research 31 (1994) 221-241. doi:10.1016/0143-974X(94)90011-6

[6] J. Jönsson, Distortional theory of thin-walled beams, ThinWalled Structures 33 (1999) 269-303. doi:10.1016/ S0263-8231 (98)00050-0

[7] N. Silvestre, D. Camotim, First-order generalised beam theory for arbitrary orthotropic materials, Thin-Walled Structures 40 (2002) 755-789. doi:10.1016/S0263-8231(02)00025-3

[8] N. Silvestre, D. Camotim, Nonlinear generalized beam theory for cold-formed steel members, International Journal of Structural Stability and Dynamics 3 (4) (2003) 461-490. doi: 10.1142/S0219455403001002.

[9] R. Bebiano, R. Gonçalves, D. Camotim, A cross-section analysis procedure to rationalise and automate the performance of gbtbased structural analyses, Thin-Walled Structures 92 (2015) 2947. doi:10.1016/j.tws.2015.02.017

[10] N. Peres, R. Gonçalves, D. Camotim, A cross-section analysis procedure to rationalise and automate the performance of gbtbased structural analyses, Thin-Walled Structures 127 (2018) 769-780. doi:10.1016/j.tws.2018.03.008.

[11] S. Ádány, B. W. Schafer, A full modal decomposition of thinwalled, single-branched open cross-section members via the constrained finite strip method, Journal of Constructional Steel Research 64 (1) (2008) 12-29. doi:10.1016/j.jcsr.2007.04.004.

[12] S. Ádány, N. Silvestre, B. W. Schafer, D. Camotim, GBT and 

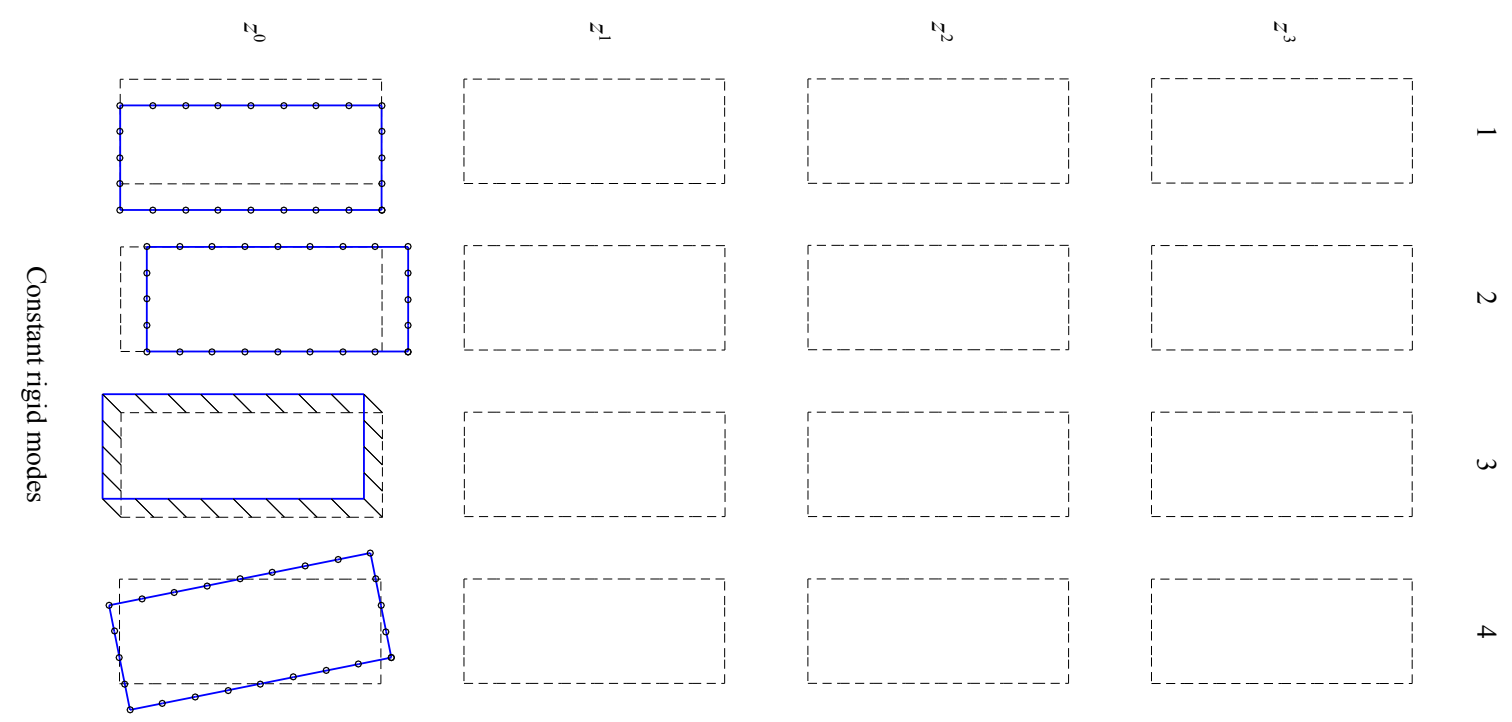

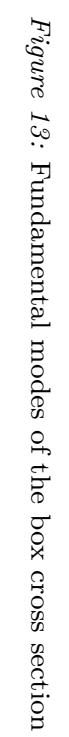
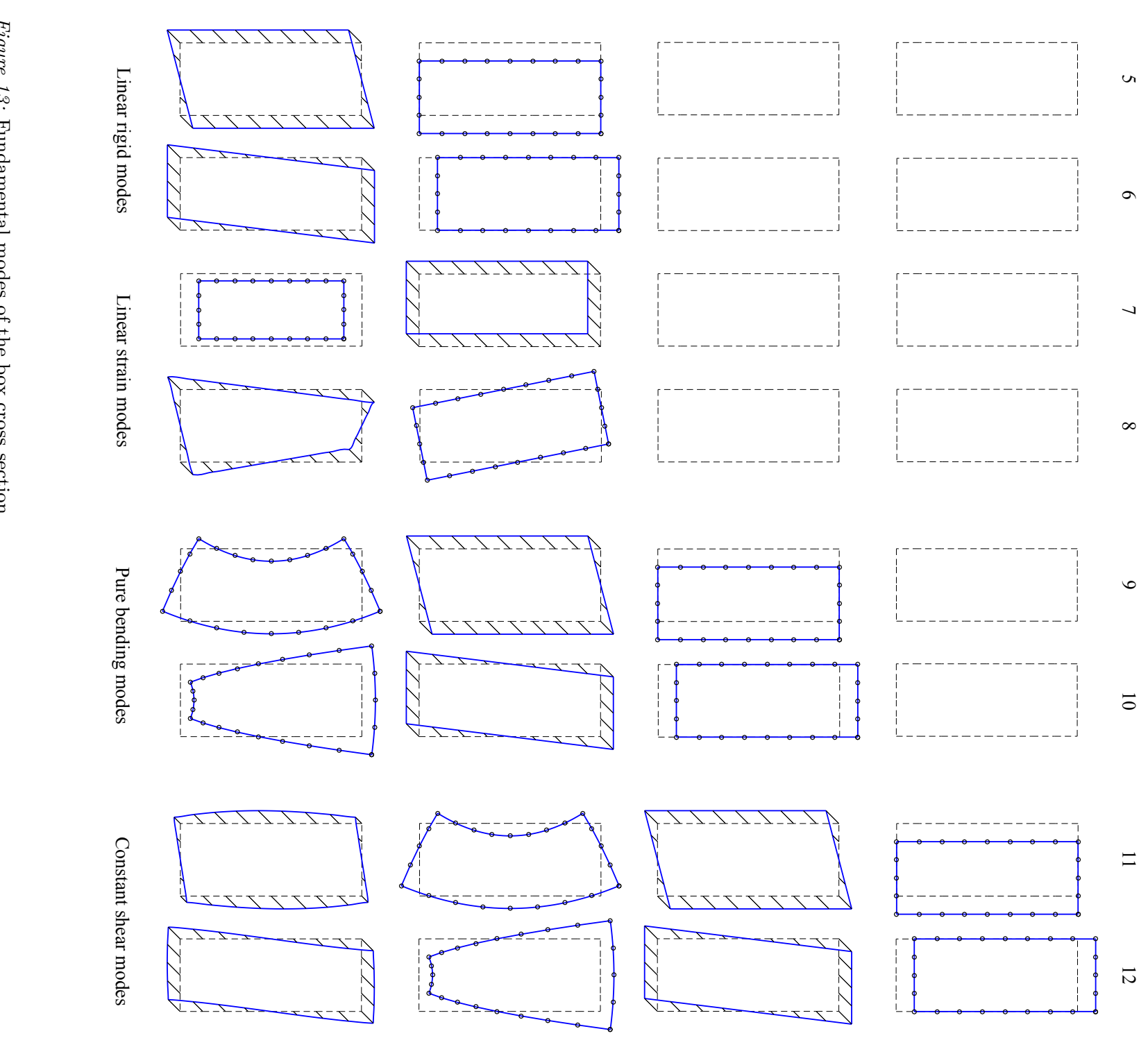

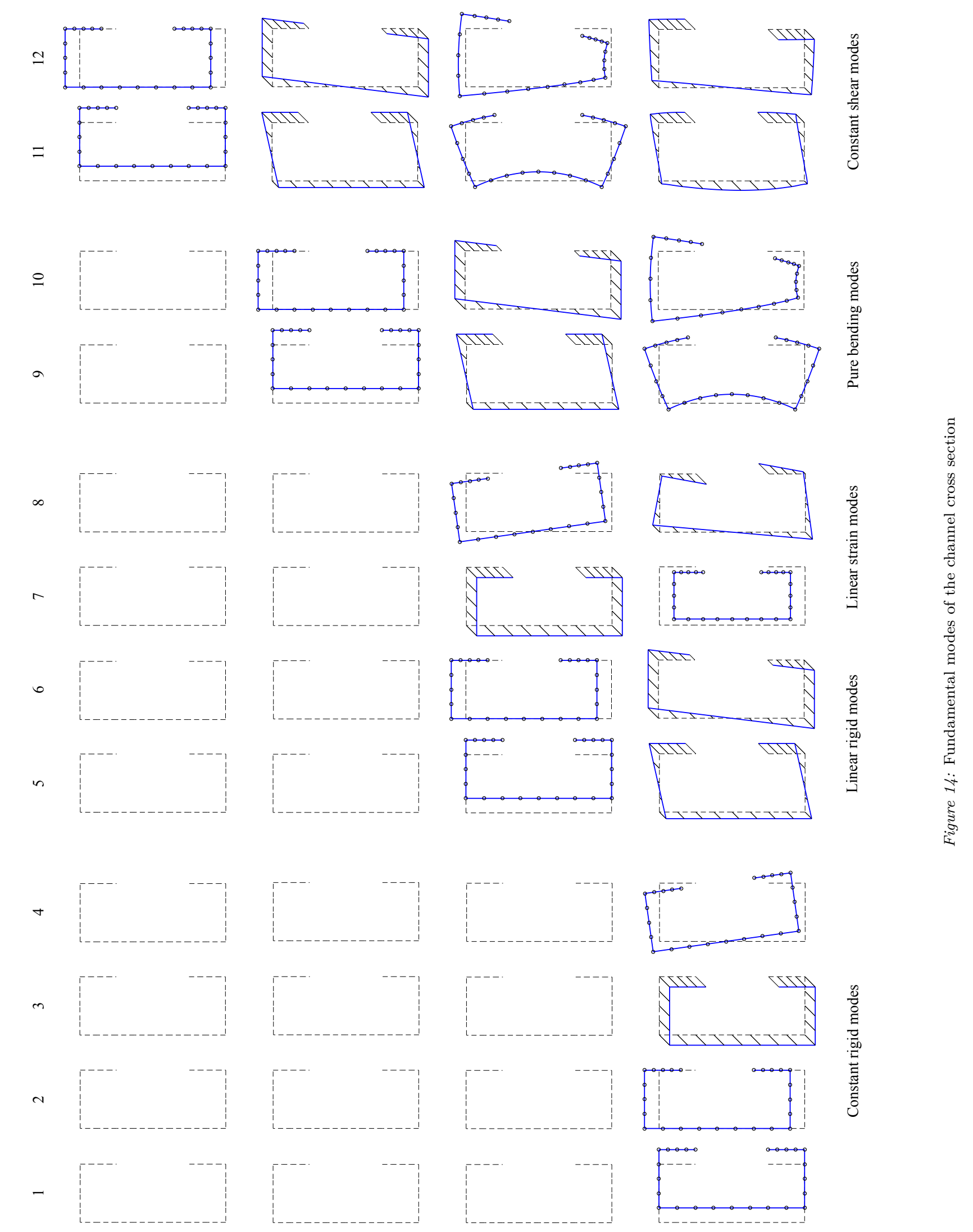

iv
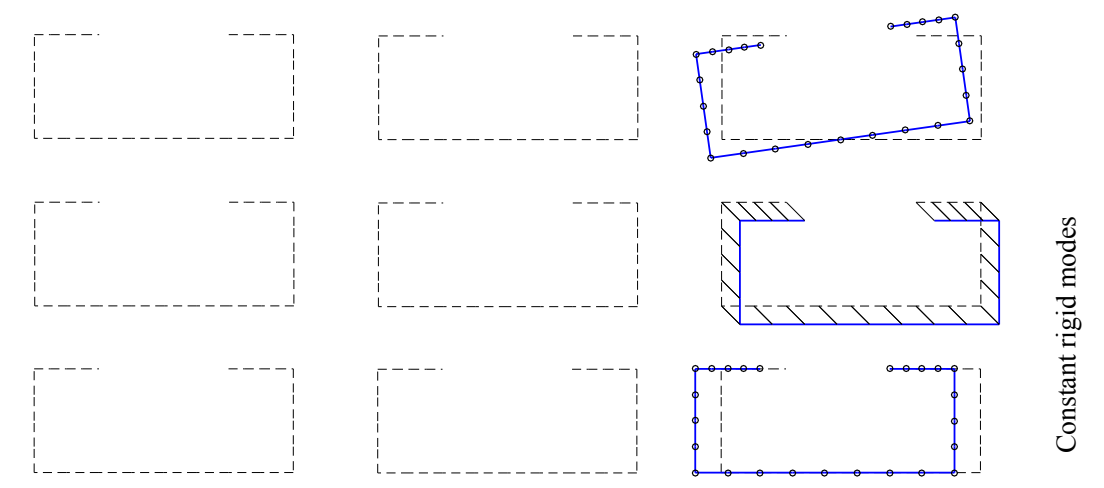
cFSM: Two modal approaches to the buckling analysis of unbranched thin-walled members, Advanced Steel Construction 5 (2) (2009) 195-223.

[13] S. Ádány, Modal identification of thin-walled members with and without holes using CFEM, Proceedings of the Eighths International Conference on Thin-Walled Structures, ICTWS 2018.

[14] J. Jönsson, M. J. Andreassen, Distortional eigenmodes and homogeneous solutions for semi-discretized thin-walled beams, Thin-Walled Structures 49 (2011) 691-707. doi:10.1016/j. tws.2010.12.009.

[15] M. Morandini, M. Chierichetti, P. Mantegazza, Characteristic behavior of prismatic anisotropic beam via generalized eigenvectors, International Journal of Solids and Structures 47 (2010) 1327-1337. doi:10.1016/j.ijsolstr.2010.01.017.

[16] R. F. Vieira, F. B. Virtuoso, E. B. R. Pereira, A higher order model for thin-walled structures with deformable crosssections, International Journal of Solids and Structures (2014) 575-598doi:10.1016/j.ijsolstr.2013.10.023

[17] S. de Miranda, A. Gutiérrez, R. Miletta, F. Ubertini, A generalized beam theory with shear deformation, Thin-Walled Structures 67 (2013) 88-100. doi:10.1016/j.tws.2013.02.012

[18] S. de Miranda, A. Madeo, R. Miletta, F. Ubertini, On the relationship of the shear deformable generalized beam theory with classical and non-classical theories, International Journal of Solids and Structures 51 (2014) 3698-3709. doi:10.1016//j . ijsolstr.2014.07.001

[19] A. B. Hansen, J. Jönsson, A gbt-framework towards modal modelling of steel structures, John Wiley \& Sons, Ltd - ce/papers (2017) 1822-1830 doi:10.1002/cepa.226

[20] R. D. Cook, D. S. Malkus, M. E. Plesha, Concepts and applications of finite element analysis, 3rd Edition, John Wiley \& Son, NewYork Chichester Brisbane Toronto Singapore, 1989.

[21] F. Tisseur, K. Meerbergen, The quadratic eigenvalue problem, Society for Industrial and Applied Mathematics 43 (2) (2001) 235-286. doi:10.1.1.32.9042

[22] Matlab, Matlab ${ }^{\circledR}$ and Simulink ${ }^{\circledR}$ used for technical computing, 2016, MAtLAB - () 1984-2016 The MathWorks, Inc., Version $2016 a$.

[23] R. J. de Figueiredo Mendes Vieira, A higher order thin-walled beam model, Ph.D. thesis, Universidade Técnica de Lisboa Instituto Superior Técnico (2010). 